\title{
Measurement of the $p \bar{p} \rightarrow t \bar{t}$ production cross section and the top quark mass at $\sqrt{s}=1.96 \mathrm{TeV}$ in the all-hadronic decay mode
}

T. Aaltonen, ${ }^{23}$ A. Abulencia, ${ }^{24}$ J. Adelman, ${ }^{13}$ T. Affolder, ${ }^{10}$ T. Akimoto, ${ }^{55}$ M. G. Albrow, ${ }^{17}$ S. Amerio,${ }^{43}$ D. Amidei, ${ }^{35}$ A. Anastassov, ${ }^{52}$ K. Anikeev, ${ }^{17}$ A. Annovi, ${ }^{19}$ J. Antos,${ }^{14}$ M. Aoki,${ }^{55}$ G. Apollinari, ${ }^{17}$ T. Arisawa, ${ }^{57}$ A. Artikov, ${ }^{15}$ W. Ashmanskas, ${ }^{17}$ A. Attal, ${ }^{3}$ A. Aurisano, ${ }^{53}$ F. Azfar, ${ }^{42}$ P. Azzi-Bacchetta, ${ }^{43}$ P. Azzurri, ${ }^{46}$ N. Bacchetta, ${ }^{43}$ W. Badgett, ${ }^{17}$ A. Barbaro-Galtieri, ${ }^{29}$ V.E. Barnes, ${ }^{48}$ B. A. Barnett, ${ }^{25}$ S. Baroiant, ${ }^{7}$ V. Bartsch, ${ }^{31}$ G. Bauer, ${ }^{33}$ P.-H. Beauchemin, ${ }^{34}$ F. Bedeschi, ${ }^{46}$ S. Behari, ${ }^{25}$ G. Bellettini, ${ }^{46}$ J. Bellinger ${ }^{59}$ A. Belloni, ${ }^{33}$ D. Benjamin, ${ }^{16}$ A. Beretvas, ${ }^{17}$ J. Beringer, ${ }^{29}$ T. Berry, ${ }^{30}$ A. Bhatti, ${ }^{50}$ M. Binkley, ${ }^{17}$ D. Bisello, ${ }^{43}$ I. Bizjak, ${ }^{31}$ R. E. Blair, ${ }^{2}$ C. Blocker, ${ }^{6}$ B. Blumenfeld,${ }^{25}$ A. Bocci, ${ }^{16}$ A. Bodek ${ }^{49}$ V. Boisvert, ${ }^{49}$ G. Bolla ${ }^{48}$ A. Bolshov, ${ }^{33}$ D. Bortoletto, ${ }^{48}$ J. Boudreau ${ }^{47}$ A. Boveia, ${ }^{10}$ B. Brau, ${ }^{10}$ L. Brigliadori, ${ }^{5}$ C. Bromberg, ${ }^{36}$ E. Brubaker, ${ }^{13}$ J. Budagov, ${ }^{15}$ H. S. Budd, ${ }^{49}$ S. Budd,${ }^{24}$ K. Burkett, ${ }^{17}$ G. Busetto, ${ }^{43}$ P. Bussey, ${ }^{21}$ A. Buzatu, ${ }^{34}$ K. L. Byrum, ${ }^{2}$ S. Cabrera,${ }^{16, q}$ M. Campanelli, ${ }^{20}$ M. Campbell, ${ }^{35}$ F. Canelli, ${ }^{17}$ A. Canepa ${ }^{45}$ S. Carrillo, ${ }^{18, i}$ D. Carlsmith, ${ }^{59}$ R. Carosi, ${ }^{46}$ S. Carron, ${ }^{34}$ B. Casal, ${ }^{11}$ M. Casarsa,${ }^{54}$ A. Castro, ${ }^{5}$ P. Catastini, ${ }^{46}$ D. Cauz, ${ }^{54}$ M. Cavalli-Sforza ${ }^{3}$ A. Cerri, ${ }^{29}$ L. Cerrito, ${ }^{31, \mathrm{~m}}$ S. H. Chang, ${ }^{28}$ Y. C. Chen, ${ }^{1}$ M. Chertok, ${ }^{7}$ G. Chiarelli, ${ }^{46}$ G. Chlachidze, ${ }^{17}$ F. Chlebana, ${ }^{17}$ I. Cho, ${ }^{28}$ K. Cho, ${ }^{28}$ D. Chokheli, ${ }^{15}$ J. P. Chou, ${ }^{22}$ G. Choudalakis, ${ }^{33}$ S. H. Chuang, ${ }^{52}$ K. Chung, ${ }^{12}$ W. H. Chung, ${ }^{59}$ Y. S. Chung, ${ }^{49}$ M. Cilijak, ${ }^{46}$ C. I. Ciobanu, ${ }^{24}$ M. A. Ciocci, ${ }^{46}$ A. Clark, ${ }^{20}$ D. Clark, ${ }^{6}$ M. Coca, ${ }^{16}$ G. Compostella, ${ }^{43}$ M.E. Convery, ${ }^{50}$ J. Conway, ${ }^{7}$ B. Cooper,${ }^{31}$ K. Copic, ${ }^{35}$ M. Cordelli, ${ }^{19}$ G. Cortiana, ${ }^{43}$ F. Crescioli, ${ }^{46}$ C. Cuenca Almenar, ${ }^{7, q}$ J. Cuevas, ${ }^{11,1}$ R. Culbertson, ${ }^{17}$ J. C. Cully, ${ }^{35}$ S. DaRonco, ${ }^{43}$ M. Datta, ${ }^{17}$ S. D'Auria, ${ }^{21}$ T. Davies, ${ }^{21}$ D. Dagenhart ${ }^{17}$ P. de Barbaro, ${ }^{49}$ S. De Cecco, ${ }^{51}$ A. Deisher, ${ }^{29}$ G. De Lentdecker, ${ }^{49, c}$ G. De Lorenzo, ${ }^{3}$ M. Dell'Orso, ${ }^{46}$ F. Delli Paoli, ${ }^{43}$ L. Demortier, ${ }^{50}$ J. Deng, ${ }^{16}$ M. Deninno, ${ }^{5}$ D. De Pedis,${ }^{51}$ P. F. Derwent, ${ }^{17}$ G. P. Di Giovanni,${ }^{44}$ C. Dionisi,${ }^{51}$ B. Di Ruzza, ${ }^{54}$ J. R. Dittmann, ${ }^{4}$ M. D'Onofrio, ${ }^{3}$ C. Dörr, ${ }^{26}$ S. Donati, ${ }^{46}$ P. Dong, ${ }^{8}$ J. Donini, ${ }^{43}$ T. Dorigo ${ }^{43}$ S. Dube, ${ }^{52}$ J. Efron, ${ }^{39}$ R. Erbacher, ${ }^{7}$ D. Errede, ${ }^{24}$ S. Errede, ${ }^{24}$ R. Eusebi, ${ }^{17}$ H. C. Fang ${ }^{29}$ S. Farrington, ${ }^{30}$ I. Fedorko, ${ }^{46}$ W. T. Fedorko, ${ }^{13}$

R. G. Feild ${ }^{60}$ M. Feindt, ${ }^{26}$ J. P. Fernandez, ${ }^{32}$ R. Field, ${ }^{18}$ G. Flanagan,${ }^{48}$ R. Forrest, ${ }^{7}$ S. Forrester, ${ }^{7}$ M. Franklin, ${ }^{22}$

J. C. Freeman, ${ }^{29}$ I. Furic, ${ }^{13}$ M. Gallinaro, ${ }^{50}$ J. Galyardt, ${ }^{12}$ J. E. Garcia, ${ }^{46}$ F. Garberson, ${ }^{10}$ A. F. Garfinkel, ${ }^{48}$ C. Gay, ${ }^{60}$ H. Gerberich, ${ }^{24}$ D. Gerdes, ${ }^{35}$ S. Giagu, ${ }^{51}$ P. Giannetti, ${ }^{46}$ K. Gibson, ${ }^{47}$ J. L. Gimmell, ${ }^{49}$ C. Ginsburg, ${ }^{17}$ N. Giokaris, ${ }^{15, a}$ M. Giordani, ${ }^{54}$ P. Giromini, ${ }^{19}$ M. Giunta, ${ }^{46}$ G. Giurgiu, ${ }^{25}$ V. Glagolev, ${ }^{15}$ D. Glenzinski, ${ }^{17}$ M. Gold, ${ }^{37}$ N. Goldschmidt, ${ }^{18}$ J. Goldstein, ${ }^{42, b}$ A. Golossanov, ${ }^{17}$ G. Gomez, ${ }^{11}$ G. Gomez-Ceballos, ${ }^{33}$ M. Goncharov, ${ }^{53}$ O. González, ${ }^{32}$ I. Gorelov ${ }^{37}$

A. T. Goshaw, ${ }^{16}$ K. Goulianos, ${ }^{50}$ A. Gresele, ${ }^{43}$ S. Grinstein,${ }^{22}$ C. Grosso-Pilcher, ${ }^{13}$ R. C. Group,${ }^{17}$ U. Grundler,${ }^{24}$

J. Guimaraes da Costa, ${ }^{22}$ Z. Gunay-Unalan, ${ }^{36}$ C. Haber, ${ }^{29}$ K. Hahn, ${ }^{33}$ S. R. Hahn, ${ }^{17}$ E. Halkiadakis,${ }^{52}$ A. Hamilton, ${ }^{20}$ B.-Y. Han ${ }^{49}$ J. Y. Han,${ }^{49}$ R. Handler, ${ }^{59}$ F. Happacher, ${ }^{19}$ K. Hara, ${ }^{55}$ D. Hare,${ }^{52}$ M. Hare ${ }^{56}$ S. Harper, ${ }^{42}$ R. F. Harr, ${ }^{58}$ R. M. Harris ${ }^{17}$ M. Hartz,${ }^{47}$ K. Hatakeyama,${ }^{50}$ J. Hauser, ${ }^{8}$ C. Hays,${ }^{42}$ M. Heck, ${ }^{26}$ A. Heijboer, ${ }^{45}$ B. Heinemann, ${ }^{29}$ J. Heinrich, ${ }^{45}$ C. Henderson, ${ }^{33}$ M. Herndon, ${ }^{59}$ J. Heuser, ${ }^{26}$ D. Hidas, ${ }^{16}$ C. S. Hill, ${ }^{10, b}$ D. Hirschbuehl, ${ }^{26}$ A. Hocker, ${ }^{17}$ A. Holloway, ${ }^{22}$ S. Hou, ${ }^{1}$ M. Houlden, ${ }^{30}$ S.-C. Hsu, ${ }^{9}$ B. T. Huffman, ${ }^{42}$ R. E. Hughes, ${ }^{39}$ U. Husemann, ${ }^{60}$ J. Huston, ${ }^{36}$ J. Incandela, ${ }^{10}$ G. Introzzi, ${ }^{46}$ M. Iori,${ }^{51}$ A. Ivanov, ${ }^{7}$ B. Iyutin,${ }^{33}$ E. James, ${ }^{17}$ D. Jang, ${ }^{52}$ B. Jayatilaka, ${ }^{16}$ D. Jeans, ${ }^{51}$ E. J. Jeon, ${ }^{28}$ S. Jindariani, ${ }^{18}$ W. Johnson, ${ }^{7}$ M. Jones,${ }^{48}$ K. K. Joo, ${ }^{28}$ S. Y. Jun, ${ }^{12}$ J. E. Jung, ${ }^{28}$ T. R. Junk,${ }^{24}$ T. Kamon,${ }^{53}$

P. E. Karchin, ${ }^{58}$ Y. Kato, ${ }^{41}$ Y. Kemp,${ }^{26}$ R. Kephart, ${ }^{17}$ U. Kerzel,,${ }^{26}$ V. Khotilovich, ${ }^{53}$ B. Kilminster, ${ }^{39}$ D. H. Kim, ${ }^{28}$ H. S. Kim, ${ }^{28}$ J.E. Kim, ${ }^{28}$ M. J. Kim, ${ }^{17}$ S. B. Kim ${ }^{28}$ S. H. Kim ${ }^{55}$ Y. K. Kim,${ }^{13}$ N. Kimura, ${ }^{55}$ L. Kirsch, ${ }^{6}$ S. Klimenko, ${ }^{18}$ M. Klute, ${ }^{33}$ B. Knuteson, ${ }^{33}$ B. R. Ko, ${ }^{16}$ K. Kondo, ${ }^{57}$ D. J. Kong, ${ }^{28}$ J. Konigsberg, ${ }^{18}$ A. Korytov, ${ }^{18}$ A. V. Kotwal, ${ }^{16}$ A. C. Kraan,${ }^{45}$ J. Kraus, ${ }^{24}$ M. Kreps,${ }^{26}$ J. Kroll, ${ }^{45}$ N. Krumnack, ${ }^{4}$ M. Kruse,,${ }^{16}$ V. Krutelyov, ${ }^{10}$ T. Kubo, ${ }^{55}$ S. E. Kuhlmann, ${ }^{2}$

T. Kuhr, ${ }^{26}$ N. P. Kulkarni, ${ }^{58}$ Y. Kusakabe, ${ }^{57}$ S. Kwang, ${ }^{13}$ A. T. Laasanen, ${ }^{48}$ S. Lai, ${ }^{34}$ S. Lami, ${ }^{46}$ S. Lammel, ${ }^{17}$ M. Lancaster, ${ }^{31}$ R. L. Lander, ${ }^{7}$ K. Lannon, ${ }^{39}$ A. Lath, ${ }^{52}$ G. Latino, ${ }^{46}$ I. Lazzizzera, ${ }^{43}$ T. LeCompte, ${ }^{2}$ J. Lee, ${ }^{49}$ J. Lee, ${ }^{28}$ Y. J. Lee, ${ }^{28}$ S. W. Lee, ${ }^{53, o}$ R. Lefèvre, ${ }^{20}$ N. Leonardo, ${ }^{33}$ S. Leone, ${ }^{46}$ S. Levy, ${ }^{13}$ J. D. Lewis, ${ }^{17}$ C. Lin,${ }^{60}$ C. S. Lin, ${ }^{17}$ M. Lindgren, ${ }^{17}$ E. Lipeles, ${ }^{9}$ A. Lister, ${ }^{7}$ D. O. Litvintsev, ${ }^{17}$ T. Liu, ${ }^{17}$ N. S. Lockyer, ${ }^{45}$ A. Loginov, ${ }^{60}$ M. Loreti, ${ }^{43}$ R.-S. Lu, ${ }^{1}$

D. Lucchesi, ${ }^{43}$ P. Lujan, ${ }^{29}$ P. Lukens, ${ }^{17}$ G. Lungu, ${ }^{18}$ L. Lyons, ${ }^{42}$ J. Lys,${ }^{29}$ R. Lysak, ${ }^{14}$ E. Lytken, ${ }^{48}$ P. Mack, ${ }^{26}$

D. MacQueen, ${ }^{34}$ R. Madrak, ${ }^{17}$ K. Maeshima, ${ }^{17}$ K. Makhoul, ${ }^{33}$ T. Maki, ${ }^{23}$ P. Maksimovic, ${ }^{25}$ S. Malde, ${ }^{42}$ S. Malik, ${ }^{31}$ G. Manca, ${ }^{30}$ A. Manousakis, ${ }^{15}$,a F. Margaroli, ${ }^{5}$ R. Marginean, ${ }^{17}$ C. Marino, ${ }^{26}$ C. P. Marino, ${ }^{24}$ A. Martin, ${ }^{60}$ M. Martin, ${ }^{25}$ V. Martin, ${ }^{21, g}$ M. Martínez, ${ }^{3}$ R. Martínez-Ballarín, ${ }^{32}$ T. Maruyama, ${ }^{55}$ P. Mastrandrea, ${ }^{51}$ T. Masubuchi, ${ }^{55}$ H. Matsunaga ${ }^{55}$ M.E. Mattson,${ }^{58}$ R. Mazini, ${ }^{34}$ P. Mazzanti, ${ }^{5}$ K. S. McFarland, ${ }^{49}$ P. McIntyre,${ }^{53}$ R. McNulty,${ }^{30, f}$ A. Mehta,${ }^{30}$ P. Mehtala, ${ }^{23}$ S. Menzemer, ${ }^{11, h}$ A. Menzione, ${ }^{46}$ P. Merkel, ${ }^{48}$ C. Mesropian, ${ }^{50}$ A. Messina, ${ }^{36}$ T. Miao, ${ }^{17}$ N. Miladinovic,${ }^{6}$ J. Miles, ${ }^{33}$ R. Miller, ${ }^{36}$ C. Mills,${ }^{10}$ M. Milnik, ${ }^{26}$ A. Mitra, ${ }^{1}$ G. Mitselmakher, ${ }^{18}$ A. Miyamoto, ${ }^{27}$ S. Moed, ${ }^{20}$ N. Moggi, ${ }^{5}$ B. Mohr, ${ }^{8}$ C. S. Moon, ${ }^{28}$ R. Moore,${ }^{17}$ M. Morello, ${ }^{46}$ P. Movilla Fernandez, ${ }^{29}$ J. Mülmenstädt, ${ }^{29}$ A. Mukherjee,${ }^{17}$ Th. Muller, ${ }^{26}$ 
R. Mumford ${ }^{25}$ P. Murat, ${ }^{17}$ M. Mussini, ${ }^{5}$ J. Nachtman, ${ }^{17}$ A. Nagano, ${ }^{55}$ J. Naganoma, ${ }^{57}$ K. Nakamura, ${ }^{55}$ I. Nakano, ${ }^{40}$ A. Napier ${ }^{56}$ V. Necula,${ }^{16}$ C. Neu, ${ }^{45}$ M. S. Neubauer, ${ }^{9}$ J. Nielsen, ${ }^{29, n}$ L. Nodulman, ${ }^{2}$ O. Norniella, ${ }^{3}$ E. Nurse,${ }^{31}$ S. H. Oh, ${ }^{16}$ Y.D. Oh, ${ }^{28}$ I. Oksuzian, ${ }^{18}$ T. Okusawa,${ }^{41}$ R. Oldeman, ${ }^{30}$ R. Orava ${ }^{23}$ K. Osterberg, ${ }^{23}$ C. Pagliarone, ${ }^{46}$ E. Palencia,${ }^{11}$ V. Papadimitriou, ${ }^{17}$ A. Papaikonomou, ${ }^{26}$ A. A. Paramonov, ${ }^{13}$ B. Parks, ${ }^{39}$ S. Pashapour, ${ }^{34}$ J. Patrick, ${ }^{17}$ G. Pauletta, ${ }^{54}$ M. Paulini, ${ }^{12}$ C. Paus, ${ }^{33}$ D. E. Pellett, ${ }^{7}$ A. Penzo, ${ }^{54}$ T. J. Phillips, ${ }^{16}$ G. Piacentino, ${ }^{46}$ J. Piedra, ${ }^{44}$ L. Pinera,${ }^{18}$ K. Pitts,${ }^{24}$ C. Plager, ${ }^{8}$ L. Pondrom, ${ }^{59}$ X. Portell, ${ }^{3}$ O. Poukhov,${ }^{15}$ N. Pounder, ${ }^{42}$ F. Prakoshyn, ${ }^{15}$ A. Pronko, ${ }^{17}$ J. Proudfoot,${ }^{2}$ F. Ptohos, ${ }^{19, \mathrm{e}}$ G. Punzi ${ }^{46}$ J. Pursley, ${ }^{25}$ J. Rademacker, ${ }^{42, \mathrm{~b}}$ A. Rahaman, ${ }^{47}$ V. Ramakrishnan, ${ }^{59}$ N. Ranjan, ${ }^{48}$ I. Redondo, ${ }^{32}$ B. Reisert, ${ }^{17}$ V. Rekovic,${ }^{37}$ P. Renton, ${ }^{42}$ M. Rescigno, ${ }^{51}$ S. Richter, ${ }^{26}$ F. Rimondi, ${ }^{5}$ L. Ristori, ${ }^{46}$ A. Robson, ${ }^{21}$ T. Rodrigo, ${ }^{11}$ E. Rogers, ${ }^{24}$ S. Rolli, ${ }^{56}$ R. Roser, ${ }^{17}$ M. Rossi,${ }^{54}$ R. Rossin,${ }^{10}$ P. Roy, ${ }^{34}$ A. Ruiz,${ }^{11}$ J. Russ, ${ }^{12}$ V. Rusu, ${ }^{13}$ H. Saarikko, ${ }^{23}$ A. Safonov, ${ }^{53}$ W. K. Sakumoto, ${ }^{49}$ G. Salamanna, ${ }^{51}$ O. Saltó, ${ }^{3}$ L. Santi, ${ }^{54}$ S. Sarkar, ${ }^{51}$ L. Sartori, ${ }^{46}$ K. Sato, ${ }^{17}$ P. Savard,${ }^{34}$ A. Savoy-Navarro, ${ }^{44}$ T. Scheidle, ${ }^{26}$ P. Schlabach, ${ }^{17}$ E. E. Schmidt, ${ }^{17}$ M. P. Schmidt, ${ }^{60}$ M. Schmitt, ${ }^{38}$ T. Schwarz, ${ }^{7}$ L. Scodellaro, ${ }^{11}$ A. L. Scott ${ }^{10}$ A. Scribano,${ }^{46}$ F. Scuri, ${ }^{46}$ A. Sedov ${ }^{48}$ S. Seidel,${ }^{37}$ Y. Seiya,${ }^{41}$ A. Semenov, ${ }^{15}$ L. Sexton-Kennedy, ${ }^{17}$ A. Sfyrla, ${ }^{20}$ S. Z. Shalhout, ${ }^{58}$ M. D. Shapiro, ${ }^{29}$ T. Shears, ${ }^{30}$ P. F. Shepard,${ }^{47}$ D. Sherman, ${ }^{22}$ M. Shimojima ${ }^{55, \mathrm{k}}$ M. Shochet, ${ }^{13}$ Y. Shon, ${ }^{59}$ I. Shreyber, ${ }^{20}$ A. Sidoti, ${ }^{46}$ P. Sinervo, ${ }^{34}$ A. Sisakyan, ${ }^{15}$ A. J. Slaughter, ${ }^{17}$ J. Slaunwhite, ${ }^{39}$ K. Sliwa, ${ }^{56}$ J. R. Smith, ${ }^{7}$ F. D. Snider, ${ }^{17}$ R. Snihur, ${ }^{34}$ M. Soderberg, ${ }^{35}$ A. Soha, ${ }^{7}$ S. Somalwar, ${ }^{52}$ V. Sorin, ${ }^{36}$ J. Spalding, ${ }^{17}$ F. Spinella, ${ }^{46}$ T. Spreitzer, ${ }^{34}$ P. Squillacioti, ${ }^{46}$ M. Stanitzki, ${ }^{60}$ A. Staveris-Polykalas, ${ }^{46}$ R. St. Denis, ${ }^{21}$ B. Stelzer, ${ }^{8}$ O. Stelzer-Chilton, ${ }^{42}$ D. Stentz, ${ }^{38}$ J. Strologas,${ }^{37}$ D. Stuart, ${ }^{10}$ J. S. Suh,${ }^{28}$ A. Sukhanov, ${ }^{18}$ H. Sun, ${ }^{56}$ I. Suslov, ${ }^{15}$ T. Suzuki, ${ }^{55}$ A. Taffard, ${ }^{24, p}$ R. Takashima, ${ }^{40}$ Y. Takeuchi, ${ }^{55}$ R. Tanaka, ${ }^{40}$ M. Tecchio, ${ }^{35}$ P. K. Teng, ${ }^{1}$ K. Terashi, ${ }^{50}$ J. Thom, ${ }^{17, \mathrm{~d}}$ A. S. Thompson, ${ }^{21}$ E. Thomson, ${ }^{45}$ P. Tipton, ${ }^{60}$ V. Tiwari, ${ }^{12}$ S. Tkaczyk, ${ }^{17}$ D. Toback, ${ }^{53}$ S. Tokar, ${ }^{14}$ K. Tollefson, ${ }^{36}$ T. Tomura,${ }^{55}$ D. Tonelli ${ }^{46}$ S. Torre, ${ }^{19}$ D. Torretta, ${ }^{17} \mathrm{~S}$. Tourneur, ${ }^{44} \mathrm{~W}$. Trischuk ${ }^{34} \mathrm{~S}$. Tsuno, ${ }^{40} \mathrm{Y}$. Tu, ${ }^{45}$ N. Turini, ${ }^{46}$ F. Ukegawa, ${ }^{55}$ S. Uozumi, ${ }^{55}$ S. Vallecorsa, ${ }^{20}$ N. van Remortel,${ }^{23}$ A. Varganov, ${ }^{35}$ E. Vataga,${ }^{37}$ F. Vazquez, ${ }^{18, i}$ G. Velev, ${ }^{17}$ C. Vellidis, ${ }^{46, a}$ G. Veramendi, ${ }^{24}$ V. Veszpremi, ${ }^{48}$ M. Vidal,${ }^{32}$ R. Vidal,${ }^{17}$ I. Vila, ${ }^{11}$ R. Vilar, ${ }^{11}$ T. Vine, ${ }^{31}$ M. Vogel, ${ }^{37}$ I. Vollrath, ${ }^{34}$ I. Volobouev, ${ }^{29, o}$ G. Volpi,${ }^{46}$ F. Würthwein,,${ }^{9}$ P. Wagner, ${ }^{53}$ R. G. Wagner, ${ }^{2}$ R. L. Wagner, ${ }^{17}$ J. Wagner, ${ }^{26}$ W. Wagner, ${ }^{26}$ R. Wallny, ${ }^{8}$ S. M. Wang, ${ }^{1}$ A. Warburton, ${ }^{34}$ D. Waters, ${ }^{31}$ M. Weinberger, ${ }^{53}$ W. C. Wester III, ${ }^{17}$ B. Whitehouse, ${ }^{56}$ D. Whiteson, ${ }^{45, p}$ A. B. Wicklund,${ }^{2}$ E. Wicklund, ${ }^{17}$ G. Williams, ${ }^{34}$ H. H. Williams, ${ }^{45}$ P. Wilson, ${ }^{17}$ B. L. Winer,${ }^{39}$ P. Wittich,${ }^{17, d}$ S. Wolbers,${ }^{17}$ C. Wolfe, ${ }^{13}$ T. Wright,${ }^{35}$ X. Wu ${ }^{20}$ S. M. Wynne, ${ }^{30}$ A. Yagil, ${ }^{9}$ K. Yamamoto, ${ }^{41}$ J. Yamaoka, ${ }^{52}$ T. Yamashita, ${ }^{40}$ C. Yang, ${ }^{60}$ U. K. Yang,,${ }^{13, j}$ Y. C. Yang, ${ }^{28}$ W. M. Yao,${ }^{29}$ G. P. Yeh,${ }^{17}$ J. Yoh, ${ }^{17}$ K. Yorita, ${ }^{13}$ T. Yoshida, ${ }^{41}$ G. B. Yu, ${ }^{49}$ I. Yu, ${ }^{28}$ S. S. Yu, ${ }^{17}$ J. C. Yun, ${ }^{17}$ L. Zanello, ${ }^{51}$ A. Zanetti, ${ }^{54}$ I. Zaw, ${ }^{22}$ X. Zhang, ${ }^{24}$ J. Zhou, ${ }^{52}$ and S. Zucchelli ${ }^{5}$

(CDF Collaboration)

\author{
${ }^{1}$ Institute of Physics, Academia Sinica, Taipei, Taiwan 11529, Republic of China \\ ${ }^{2}$ Argonne National Laboratory, Argonne, Illinois 60439, USA \\ ${ }^{3}$ Institut de Fisica d'Altes Energies, Universitat Autonoma de Barcelona, E-08193, Bellaterra (Barcelona), Spain \\ ${ }^{4}$ Baylor University, Waco, Texas 76798, USA \\ ${ }^{5}$ Istituto Nazionale di Fisica Nucleare, University of Bologna, I-40127 Bologna, Italy \\ ${ }^{6}$ Brandeis University, Waltham, Massachusetts 02254, USA \\ ${ }^{7}$ University of California, Davis, Davis, California 95616, USA \\ ${ }^{8}$ University of California, Los Angeles, Los Angeles, California 90024, USA \\ ${ }^{9}$ University of California, San Diego, La Jolla, California 92093, USA \\ ${ }^{10}$ University of California, Santa Barbara, Santa Barbara, California 93106, USA \\ ${ }^{11}$ Instituto de Fisica de Cantabria, CSIC-University of Cantabria, 39005 Santander, Spain \\ ${ }^{12}$ Carnegie Mellon University, Pittsburgh, Pennsylvania 15213, USA \\ ${ }^{13}$ Enrico Fermi Institute, University of Chicago, Chicago, Illinois 60637, USA \\ ${ }^{14}$ Comenius University, 84248 Bratislava, Slovakia and Institute of Experimental Physics, 04001 Kosice, Slovakia \\ ${ }^{15}$ Joint Institute for Nuclear Research, RU-141980 Dubna, Russia \\ ${ }^{16}$ Duke University, Durham, North Carolina 27708 \\ ${ }^{17}$ Fermi National Accelerator Laboratory, Batavia, Illinois 60510, USA \\ ${ }^{18}$ University of Florida, Gainesville, Florida 32611, USA \\ ${ }^{19}$ Laboratori Nazionali di Frascati, Istituto Nazionale di Fisica Nucleare, I-00044 Frascati, Italy \\ ${ }^{20}$ University of Geneva, CH-1211 Geneva 4, Switzerland \\ ${ }^{21}$ Glasgow University, Glasgow G12 8QQ, United Kingdom \\ ${ }^{22}$ Harvard University, Cambridge, Massachusetts 02138, USA
}


${ }^{23}$ Division of High Energy Physics, Department of Physics, University of Helsinki and Helsinki Institute of Physics, FIN-00014, Helsinki, Finland

${ }^{24}$ University of Illinois, Urbana, Illinois 61801, USA

${ }^{25}$ The Johns Hopkins University, Baltimore, Maryland 21218, USA

${ }^{26}$ Institut für Experimentelle Kernphysik, Universität Karlsruhe, 76128 Karlsruhe, Germany

${ }^{27}$ High Energy Accelerator Research Organization (KEK), Tsukuba, Ibaraki 305, Japan

${ }^{28}$ Center for High Energy Physics, Kyungpook National University, Taegu 702-701, Korea; Seoul National University, Seoul 151-742, Korea; and SungKyunKwan University, Suwon 440-746, Korea

${ }^{29}$ Ernest Orlando Lawrence Berkeley National Laboratory, Berkeley, California 94720, USA

${ }^{30}$ University of Liverpool, Liverpool L69 7ZE, United Kingdom

${ }^{31}$ University College London, London WC1E 6BT, United Kingdom

${ }^{32}$ Centro de Investigaciones Energeticas Medioambientales y Tecnologicas, E-28040 Madrid, Spain

${ }^{33}$ Massachusetts Institute of Technology, Cambridge, Massachusetts 02139, USA

${ }^{34}$ Institute of Particle Physics, McGill University, Montréal, Canada H3A 2 T8 and University of Toronto, Toronto, Canada M5S 1A7

${ }^{35}$ University of Michigan, Ann Arbor, Michigan 48109, USA

${ }^{36}$ Michigan State University, East Lansing, Michigan 48824, USA

${ }^{37}$ University of New Mexico, Albuquerque, New Mexico 87131, USA

${ }^{38}$ Northwestern University, Evanston, Illinois 60208, USA

${ }^{39}$ The Ohio State University, Columbus, Ohio 43210, USA

${ }^{40}$ Okayama University, Okayama 700-8530, Japan

${ }^{41}$ Osaka City University, Osaka 588, Japan

${ }^{42}$ University of Oxford, Oxford OX1 3RH, United Kingdom

${ }^{43}$ University of Padova, Istituto Nazionale di Fisica Nucleare, Sezione di Padova-Trento, I-35131 Padova, Italy

${ }^{44}$ LPNHE, Universite Pierre et Marie Curie/IN2P3-CNRS, UMR7585, Paris, F-75252 France

${ }^{45}$ University of Pennsylvania, Philadelphia, Pennsylvania 19104, USA

${ }^{46}$ Istituto Nazionale di Fisica Nucleare Pisa, Universities of Pisa, Siena and Scuola Normale Superiore, I-56127 Pisa, Italy

${ }^{47}$ University of Pittsburgh, Pittsburgh, Pennsylvania 15260, USA

${ }^{48}$ Purdue University, West Lafayette, Indiana 47907, USA

${ }^{49}$ University of Rochester, Rochester, New York 14627, USA

${ }^{50}$ The Rockefeller University, New York, New York 10021, USA

${ }^{51}$ Istituto Nazionale di Fisica Nucleare, Sezione di Roma 1, University of Rome "La Sapienza," I-00185 Roma, Italy

${ }^{52}$ Rutgers University, Piscataway, New Jersey 08855, USA

${ }^{53}$ Texas A\&M University, College Station, Texas 77843, USA

${ }^{54}$ Istituto Nazionale di Fisica Nucleare, University of Trieste/Udine, Italy

${ }^{55}$ University of Tsukuba, Tsukuba, Ibaraki 305, Japan

${ }^{56}$ Tufts University, Medford, Massachusetts 02155, USA

${ }^{57}$ Waseda University, Tokyo 169, Japan

${ }^{58}$ Wayne State University, Detroit, Michigan 48201, USA

${ }^{\mathrm{a}}$ Visiting from University of Athens, 15784 Athens, Greece.

${ }^{\mathrm{b}}$ Visiting from University of Bristol, Bristol BS8 1TL, United Kingdom.

${ }^{\mathrm{c}}$ Visiting from University Libre de Bruxelles, B-1050 Brussels, Belgium.

${ }^{\mathrm{d}}$ Visiting from Cornell University, Ithaca, NY 14853, USA.

${ }^{\mathrm{e}}$ Visiting from University of Cyprus, Nicosia CY-1678, Cyprus.

${ }^{\mathrm{f}}$ Visiting from University College Dublin, Dublin 4, Ireland.

${ }^{\mathrm{g}}$ Visiting from University of Edinburgh, Edinburgh EH9 3JZ, United Kingdom.

${ }^{\mathrm{h}}$ Visiting from University of Heidelberg, D-69120 Heidelberg, Germany.

${ }^{\mathrm{i}}$ Visiting from Universidad Iberoamericana, Mexico D.F., Mexico.

${ }^{\mathrm{j}}$ Visiting from University of Manchester, Manchester M13 9PL, United Kingdom.

${ }^{\mathrm{k}}$ Visiting from Nagasaki Institute of Applied Science, Nagasaki, Japan.

${ }^{1}$ Visiting from University de Oviedo, E-33007 Oviedo, Spain.

${ }^{\mathrm{m}}$ Visiting from University of London, Queen Mary College, London, E1 4NS, United Kingdom.

${ }^{\mathrm{n}}$ Visiting from University of California Santa Cruz, Santa Cruz, CA 95064, USA.

${ }^{\circ}$ Visiting from Texas Tech University, Lubbock, TX 79409, USA.

${ }^{\mathrm{p}}$ Visiting from University of California, Irvine, Irvine, CA 92697, USA.

${ }^{\mathrm{q}}$ Visiting from IFIC (CSIC-Universitat de Valencia), 46071 Valencia, Spain. 


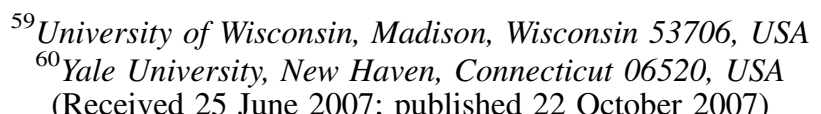

We report the measurements of the $t \bar{t}$ production cross section and of the top quark mass using $1.02 \mathrm{fb}^{-1}$ of $p \bar{p}$ data collected with the CDF II detector at the Fermilab Tevatron. We select events with six or more jets on which a number of kinematical requirements are imposed by means of a neural network algorithm. At least one of these jets must be identified as initiated by a $b$-quark candidate by the reconstruction of a secondary vertex. The cross section is measured to be $\sigma_{t \bar{t}}=8.3 \pm 1.0(\text { stat })_{-1.5}^{+2.0} \times$ (syst) \pm 0.5 (lumi) pb, which is consistent with the standard model prediction. The top quark mass of $174.0 \pm 2.2$ (stat) \pm 4.8 (syst) $\mathrm{GeV} / c^{2}$ is derived from a likelihood fit incorporating reconstructed mass distributions representative of signal and background.

DOI: 10.1103/PhysRevD.76.072009

PACS numbers: 14.65.Ha, 13.85.Ni, 13.85.Qk

\section{INTRODUCTION}

The measurement of the top quark properties allows one to verify the consistency of the standard model. At the Fermilab Tevatron Collider top quarks are produced mostly in pairs and the measurement of the $t \bar{t}$ cross section tests the next-to-leading-order QCD calculations. Moreover, accurate measurements of the top quark mass and of the mass of the $W$ boson provide constraints on the mass of the hypothetical standard model Higgs boson [1].

At the Tevatron center-of-mass energy, $\sqrt{s}=1.96 \mathrm{TeV}$, the predicted $t \bar{t}$ production cross section is $6.7 \mathrm{pb}$ [2] for an assumed top quark mass of $175 \mathrm{GeV} / c^{2}$, with a total theoretical uncertainty of approximately $15 \%$, due to the choice of renormalization and factorization scales. The top quark decays into a $W$ boson and a $b$ quark almost $100 \%$ of the time; the $W$ boson subsequently decays to either a quark-antiquark pair or a lepton-neutrino pair. The resulting final states are then usually distinguished by the number of charged energetic leptons ( $e$ or $\mu$ ) and the number of jets.

In this analysis, we examine events characterized by a multijet topology and no energetic lepton ("all-hadronic" mode). This $t \bar{t}$ final state has the advantage of a large branching ratio $(\approx 4 / 9)$ and of having no undetected top decay physical observables in the final state. In addition, discrepancies in top quark cross section and mass measurements between this and other decay channels could indicate contributions from physics beyond the standard model [3]. The major challenge of this channel is the very large QCD multijet background which dominates the signal by 3 orders of magnitude after the application of the online trigger selection. To improve the signal-tobackground ratio (S/B), requirements based on the kinematical and topological characteristics of standard model $t \bar{t}$ events are expressed in terms of a neural network and applied to the data. This neural network selection reaches a S/B of about $1 / 12$, improving the S/B by $60 \%$ with respect to the selection based on kinematical cuts used in previous analyses $[4,5]$. The neural network selection is followed by the requirement of jets identified as originating from $b$ quarks using a secondary vertex $b$-tagging algorithm, achieving a $\mathrm{S} / \mathrm{B}$ of about $1 / 2$.

The purity of the selected sample enables the clear observation of $t \bar{t}$ candidates and the measurement of the $t \bar{t}$ production cross section and of the top quark mass. Top quark events result in final states with a number of $b$-tagged jets larger than in inclusive QCD multijet production, so we use the excess of such tags to measure the $t \bar{t}$ production cross section. A reconstructed top quark mass is then determined from a kinematical fit of the six leading jets in the event to a $t \bar{t}$ final state. The distribution of reconstructed top quark masses is then compared to the distributions expected from background and $t \bar{t}$ events simulated at various values of the top quark mass, to obtain the value which best describes the data.

Given the theoretical uncertainties on the production cross section for events generated with $N$ partons at tree level, a more accurate background estimate is obtained from the data themselves ("data-driven") rather than from theoretical prediction of cross section and simulations.

The CDF and D0 Collaborations previously measured the $t \bar{t}$ production cross section and the top quark mass in the all-hadronic channel [6] using data sets with integrated luminosities of approximately $110 \mathrm{pb}^{-1}$ collected at $\sqrt{\mathrm{s}}=$ $1.8 \mathrm{TeV}$ during the Tevatron run I (1992-1996). More recent run II measurements of the cross section [4] and mass [5] have been performed by CDF using $311 \mathrm{pb}^{-1}$ collected with the CDF II detector at $\sqrt{s}=1.96 \mathrm{TeV}$ (March 2002-August 2004). The results reported here are based on the data taken between March 2002 and February 2006, corresponding to an integrated luminosity of $1.02 \mathrm{fb}^{-1}$. These measurements complement other recent $t \bar{t}$ cross section and top quark mass determinations by CDF [7-10] and D0 [11-13] in other final states.

The organization of the paper is as follows: Sec. II contains a brief description of the CDF II detector. The trigger and the neural-network-based sample selection are described in Sec. III. The $b$-tagging algorithm and its efficiency for identifying $b$ jets are described in Sec. IV. In Sec. V the data-driven method we use for estimating the 
background from experimental multijet data is applied and the related systematic uncertainties are evaluated. Section VI describes the optimization of the kinematical selection and the associated efficiency. The $t \bar{t}$ production cross section is presented in Sec. VII. The reconstruction of the top quark mass and the corresponding background and signal distributions are described in Sec. VIII; the method for fitting these distributions is discussed in Sec. IX. Section X summarizes the expected contributions to the systematic uncertainty on the top quark mass measurement, while Sec. XI describes the top quark mass measurement. Finally, cross section and mass measurements are summarized in Sec. XII.

\section{THE CDF II DETECTOR}

The CDF II detector [14] is an azimuthally and forwardbackward symmetric apparatus designed to study $p \bar{p}$ collisions at the Tevatron. A cylindrical coordinate system as described in [15] is used. The detector consists of a magnetic spectrometer surrounded by calorimeters and muon chambers. The charged particle tracking system is immersed in a $1.4 \mathrm{~T}$ solenoidal magnetic field with axis parallel to the beam line. A set of silicon microstrip detectors provides charged particle tracking in the radial range from 1.5 to $28 \mathrm{~cm}$, while a $3.1 \mathrm{~m}$ long open-cell drift chamber, the central outer tracker (COT), covers the radial range from 40 to $137 \mathrm{~cm}$. In combination the silicon and COT detectors provide excellent tracking up to pseudorapidities [15] $|\eta| \leq 1.1$, with decreasing coverage up to $|\eta| \leq 2.0$. Segmented electromagnetic and hadronic calorimeters surround the tracking system and measure the energy of interacting particles. The electromagnetic and hadronic calorimeters are lead-scintillator and ironscintillator sampling devices, respectively, covering the range $|\eta| \leq 3$.6. They are segmented in the central region $(|\eta|<1.1)$ in towers subtending $15^{\circ}$ in azimuth and 0.1 in $\eta$, and in the forward region $(1.1<|\eta|<3.6)$ in towers subtending $7.5^{\circ}$ for $|\eta|<2.11$ and $15^{\circ}$ for $|\eta|>2.11$. The electromagnetic calorimeters [16,17] are instrumented with proportional and scintillating strip detectors that measure the transverse profile of electromagnetic showers at a depth corresponding to the expected shower maximum. Drift chambers located outside the central hadronic calorimeters and behind a $60 \mathrm{~cm}$ iron shield detect muons with $|\eta| \leq 0.6$ [18]. Additional drift chambers and scintillation counters detect muons in the region $0.6<|\eta|<1.5$. Gas Cherenkov counters [19] with a coverage of $3.7<|\eta|<$ 4.7 measure the average number of inelastic $p \bar{p}$ collisions and thereby determine the luminosity.

\section{MULTIJET EVENT SELECTION}

The all-hadronic final state of $t \bar{t}$ events is characterized by the presence of at least six jets from the decay of the two top quarks. Collisions are selected in real time using a multijet trigger, relying on calorimeter information, which was specially developed to collect the events used in this analysis. Subsequently, jets are identified off-line by grouping clusters of energy in the calorimeter using a fixed-cone algorithm with a radius 0.4 in $\eta-\phi$ space [20]. The typical jet transverse energy [15] resolution is approximately $\left(0.1 \times E_{T}+1.0\right) \mathrm{GeV}$ [21], where $E_{T}$ is the jet transverse energy in $\mathrm{GeV}$. After a preliminary selection of multijet events, a neural network selection based on relevant kinematical variables is used to provide the most precise cross section and mass measurements.

\section{A. Multijet trigger}

The CDF trigger system has three levels. The first two levels consist of special-purpose electronics and the third one of conventional digital processors. For triggering purposes the calorimeter granularity is simplified to a $24 \times 24$ grid in $\eta-\phi$ space with each "trigger tower" spanning approximately $15^{\circ}$ in $\phi$ and 0.2 in $\eta$ covering one or two physical towers. At level 1 , the jet trigger requires a single tower with transverse energy $E_{T}^{\text {tow }} \geq 10 \mathrm{GeV}$. At level 2 we require the total transverse energy, summed over all the trigger towers, $\sum E_{T}^{\text {tow }}$, to be $\geq 175 \mathrm{GeV}$, and the presence of four or more clusters of calorimeter towers, each cluster with transverse energy $E_{T}^{\mathrm{cls}} \geq 15 \mathrm{GeV}$. In order to maintain an acceptable trigger rate, as the peak instantaneous luminosity increased, the $\sum E_{T}^{\text {tow }}$ threshold has been increased over the course of the data taking, $175 \mathrm{GeV}$ being the latest value. This threshold has been applied, off-line, to the whole data set. Finally, the third trigger level confirms the level 2 selection using a more accurate determination of the jet energy, requiring four or more reconstructed jets with $E_{T} \geq 10 \mathrm{GeV}$. A total of 4340 143 events satisfy the trigger requirements, with an efficiency of about $58 \%$ for inclusive $t \bar{t}$ events, and of $80 \%$ for all-hadronic $t \bar{t}$ events. The efficiency has been estimated using Monte Carlo generated $t \bar{t}$ events, described later. This corresponds to the S/B of approximately $1 / 1100$ for the theoretical cross section of $6.7 \mathrm{pb}$, where the background is represented by the multijet events themselves.

\section{B. Preselection and topology requirements}

Events satisfying the trigger requirements are reconstructed in terms of their final state observables (tracks, vertices, charged leptons, jets). We retain only those events that are well contained in detector acceptance, requiring the primary event vertex [22] to lie inside the luminous region $(|z|<60 \mathrm{~cm})$. The jet energies are corrected for detector response and multiple interactions. First, we take into account the $\eta$ dependence of detector response and energy loss in the uninstrumented regions. After a small correction for the extra energy deposited by multiple collisions in the same beam-beam bunch crossing, a correction for calorimeter nonlinearity is applied so that the jet energies correspond, on average, to the energy of all the 
particles within the jet cone. All systematic uncertainties for the individual corrections are added in quadrature to obtain the total uncertainty on the estimate of the initial parton energy. This uncertainty goes from $8 \%$ to $3 \%$ with jet transverse energy increasing from $15 \mathrm{GeV}$ to $50 \mathrm{GeV}$, and remains approximately constant at $3 \%$ above $50 \mathrm{GeV}$ [23].

For this analysis, each jet is required to have $E_{T} \geq$ $15 \mathrm{GeV}$ and $|\eta| \leq 2$ after all corrections have been applied. In order to remove the events from the $t \bar{t}$ leptonic channels, we veto events containing any well-identified high- $p_{\mathrm{T}}$ electrons and muons as defined in [8], and require that $\frac{\mathbb{Z}_{T}}{\sqrt{\sum E_{T}}}$ be $<3 \sqrt{\mathrm{GeV}}$ [9], where the missing transverse energy, $\mathbb{E}_{T}$ [24], is computed with reference to the detector origin and is corrected for any identified muons and the position of the $p \bar{p}$ collision point, while $\sum E_{T}$ is obtained by summing the $E_{T}$ 's of all the selected jets. At this stage, called "preselection," simulations show that the fraction of leptonic events amounts to about $14 \%$ of all accepted $t \bar{t}$ events. To avoid overlaps between jets we require jet pairs to be separated by at least 0.5 units in the $\eta-\phi$ space. About $3.5 \times 10^{6}$ events pass these preselection requirements (S/B 1/1000). Finally, we define the topology of the signal region by selecting events with a number of jets $6 \leq N_{\text {jets }} \leq 8$ to optimize the signal fraction. A total of 506 567 events pass this additional requirement with an expected S/B of approximately $1 / 370$, i.e. about $0.3 \%$; the remaining events with lower jet multiplicity have much smaller values of S/B and are used as control regions. The residual fraction of leptonic $t \bar{t}$ events amounts to only $5 \%$ of all accepted events.

\section{Neural-network-based kinematical selection}

In order to further improve the $\mathrm{S} / \mathrm{B}$ we use a neural network approach to recognize in more detail the features of signal and background events, including correlations between the kinematical variables which enter as input nodes in the network. We thus expect a better separation between signal and background relative to the former technique [4] where correlations were not fully considered. The network uses the MLPFIT package [25] as implemented by ROOT [26] through the TMultiLayerPerceptron class. We define a kinematical selection based on dynamical and topological properties of the candidate event. The number of variables used should allow the best possible description of the event properties, but, at the same time, too many input variables can worsen the performance, given the limited training statistics. As a guideline we studied different neural network configurations, in terms of inputs and hidden nodes, adding a few variables at a time, looking for the best performance in terms of largest S/B. The first quantities considered are those used in [4]: the total transverse energy of the jets, $\sum E_{T}$; the quantity $\sum_{3} E_{T} \equiv$ $\sum E_{T}-E_{T}^{1}-E_{T}^{2}$, obtained by removing the contribution of the two jets with the highest $E_{T}$; the centrality, defined as

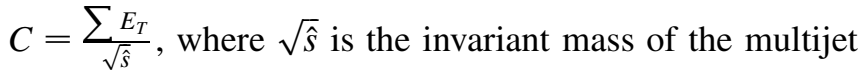
system; and the aplanarity $A$, defined as $A=\frac{3}{2} \mathcal{Q}_{1}, \mathcal{Q}_{1}$ being the smallest of the three normalized eigenvalues of the sphericity tensor, $M^{a b}=\sum_{j} P_{j}^{a} P_{j}^{b}$, calculated in the center-of-mass system of all jets, where the indices $a$ and $b$ refer to the spatial components of the jet four-momentum $P_{j}$. In addition, we consider the dynamical properties of dijet and trijet systems through the use of the minimum and maximum values of the invariant mass among all possible jet permutations: $M_{2 j}^{\min }, M_{2 j}^{\max }, M_{3 j}^{\min }$, and $M_{3 j}^{\max }$. We obtain another set of discriminating variables combining the transverse energy of the jets with their emission direction, represented by the angle $\theta^{\star}$ between the jet direction, as measured in the center-of-mass frame of all jets, and the proton beam axis. The variable $\cos \theta^{\star}$ has been shown [27] to have discriminating power against the background, and we use here the quantity $E_{T}^{\star}=E_{T} \sin ^{2} \theta^{\star}$ which tends to have larger values in the signal in comparison to the background events; this effect is enhanced for the jets with higher $E_{T}$. The variables we choose as additional inputs to the neural network are then $E_{T}^{\star, 1}$ and $E_{T}^{\star, 2}$ for the two highest- $E_{T}$ jets, and $\left\langle E_{T}^{\star}\right\rangle$ defined as the geometric mean over the remaining $\left(N_{\text {jets }}-2\right)$ jets. The 11 variables used as inputs to the neural network are summarized in Table I. Comparisons of the background-dominated data and Monte Carlo generated signal events for the 11 kinematical variables are shown in Figs. 1-5. The network is trained on same-size samples of signal and background events with $6 \leq N_{\text {jets }} \leq 8$ (about 507000 events). In order to model the signal we use the PYTHIA v6.2 [28] leadingorder Monte Carlo generator with parton showering followed by a simulation of the CDF II detector. The reference top quark mass chosen for the training is $M_{\text {top }}=175 \mathrm{GeV} / c^{2}$. The background is obtained from the multijet data events themselves, since the signal fraction at the initial stage is expected to be very small. Among

TABLE I. Input variables to the neural network.

\begin{tabular}{lc}
\hline \hline Variable & Description \\
\hline$\sum_{T} E_{T}$ & Scalar sum of the transverse energies of all jets \\
$\sum_{3} E_{T}$ & As above, except the two highest- $E_{T}$ jets \\
$A$ & Centrality \\
$M_{2 j}^{\min }$ & Aplanarity \\
$M_{2 j}^{\max }$ & Minimum dijet invariant mass \\
$M_{3 j}^{\min }$ & Maximum dijet invariant mass \\
$M_{3 j}^{\max }$ & Minimum trijet invariant mass \\
$E_{T}^{\star, 1}$ & Maximum trijet invariant mass \\
$E_{T}^{\star, 2}$ & $E_{T} \sin ^{2} \theta^{\star}$ for the highest- $E_{T}$ jet \\
$\left\langle E_{T}^{\star}\right\rangle$ & $E_{T} \sin ^{2} \theta^{\star}$ for the next-to-highest- $E_{T}$ jet \\
\hline \hline
\end{tabular}



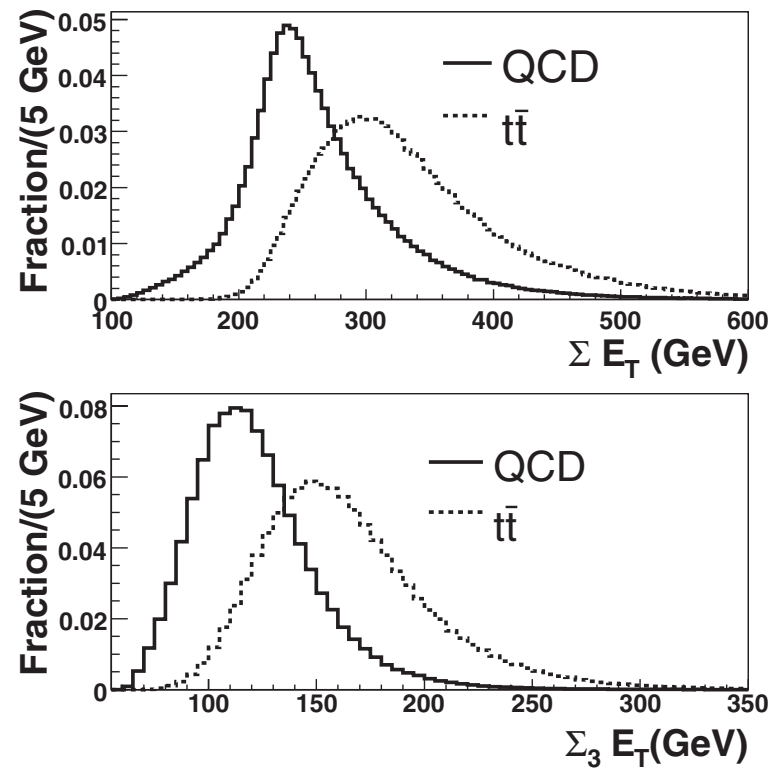

FIG. 1. $\sum E_{T}$ (top panel) and $\sum_{3} E_{T}$ (bottom panel) distributions in QCD multijet (solid histogram) and $t \bar{t}$ Monte Carlo (dashed histogram) events with $6 \leq N_{\text {jets }} \leq 8$. All histograms are normalized to unity.
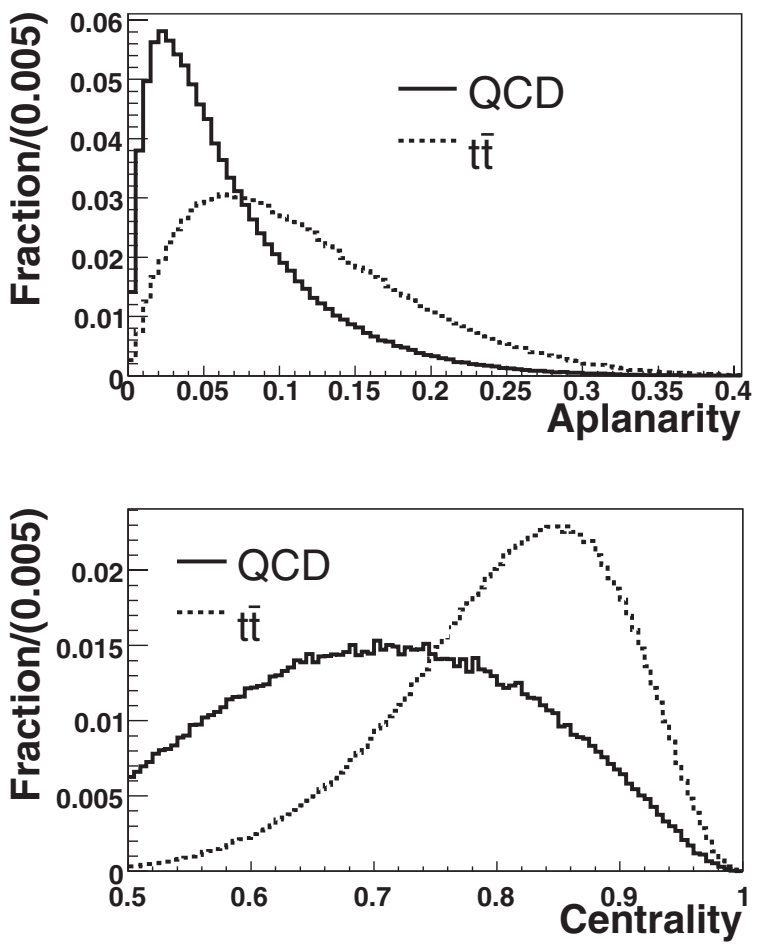

FIG. 2. Aplanarity (top panel) and centrality (bottom panel) distributions in QCD multijet (solid histogram) and $t \bar{t}$ Monte Carlo (dashed histogram) events with $6 \leq N_{\text {jets }} \leq 8$. All histograms are normalized to unity.
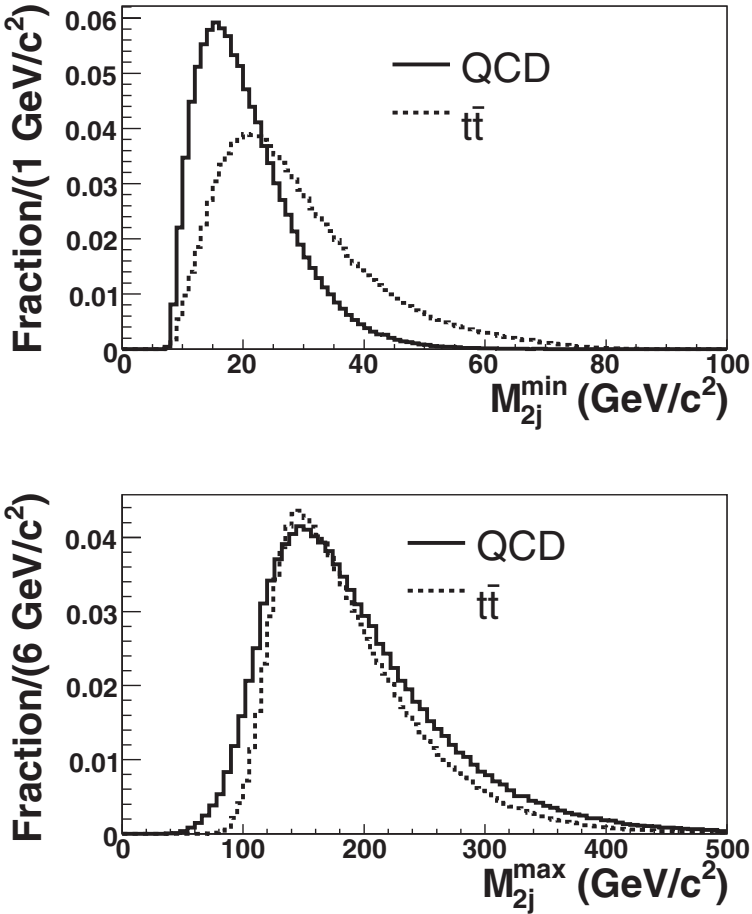

FIG. 3. $\quad M_{2 j}^{\min }$ (top panel) and $M_{2 j}^{\max }$ (bottom panel) distributions in QCD multijet (solid histogram) and $t \bar{t}$ Monte Carlo (dashed histogram) events with $6 \leq N_{\text {jets }} \leq 8$. All histograms are normalized to unity.
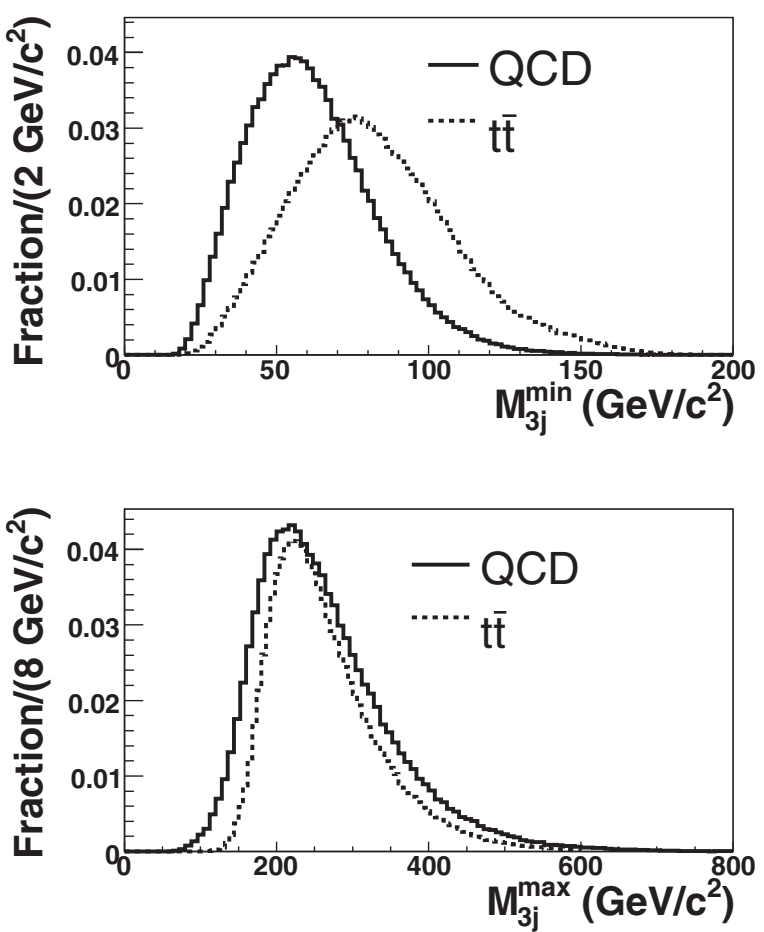

FIG. 4. $M_{3 j}^{\min }$ (top panel) and $M_{3 j}^{\max }$ (bottom panel) distributions in QCD multijet (solid histogram) and $t \bar{t}$ Monte Carlo (dashed histogram) events with $6 \leq N_{\text {jets }} \leq 8$. All histograms are normalized to unity. 

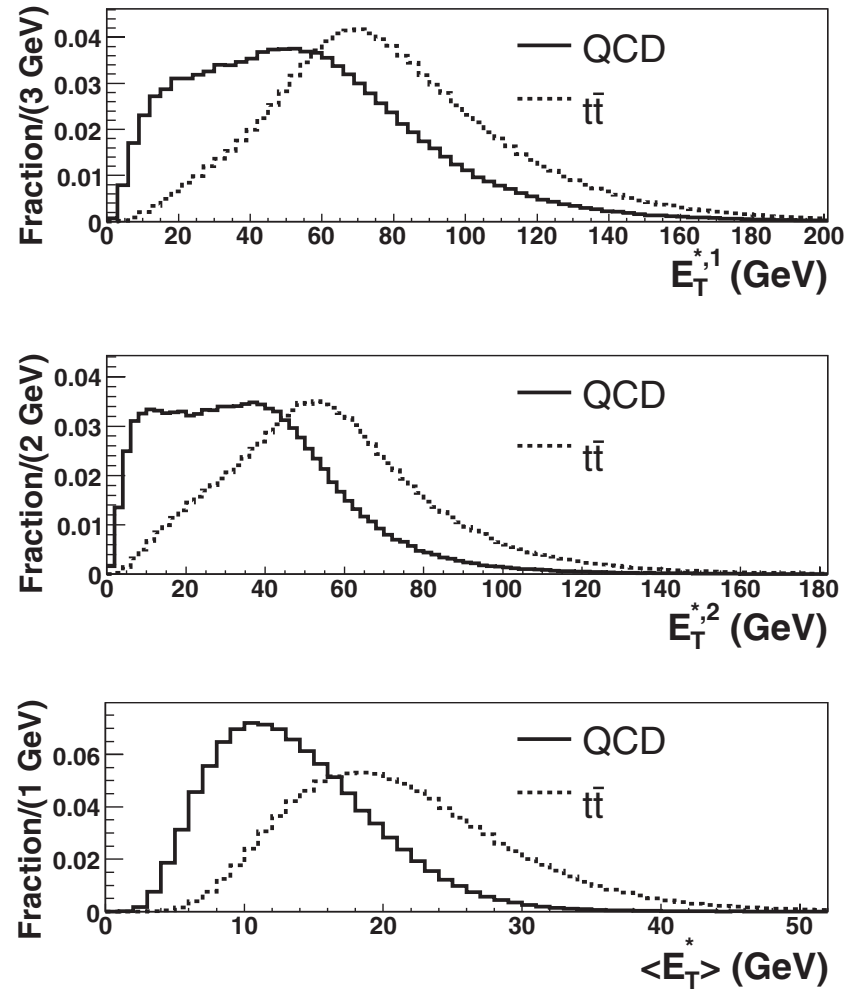

FIG. 5. Kinematical distributions in QCD multijet (solid histogram) and $t \bar{t}$ Monte Carlo (dashed histogram) events with $6 \leq$ $N_{\text {jets }} \leq 8$. From top to bottom: $E_{T}^{\star, 1}, E_{T}^{\star, 2}$, and $\left\langle E_{T}^{\star}\right\rangle$. All histograms are normalized to unity.

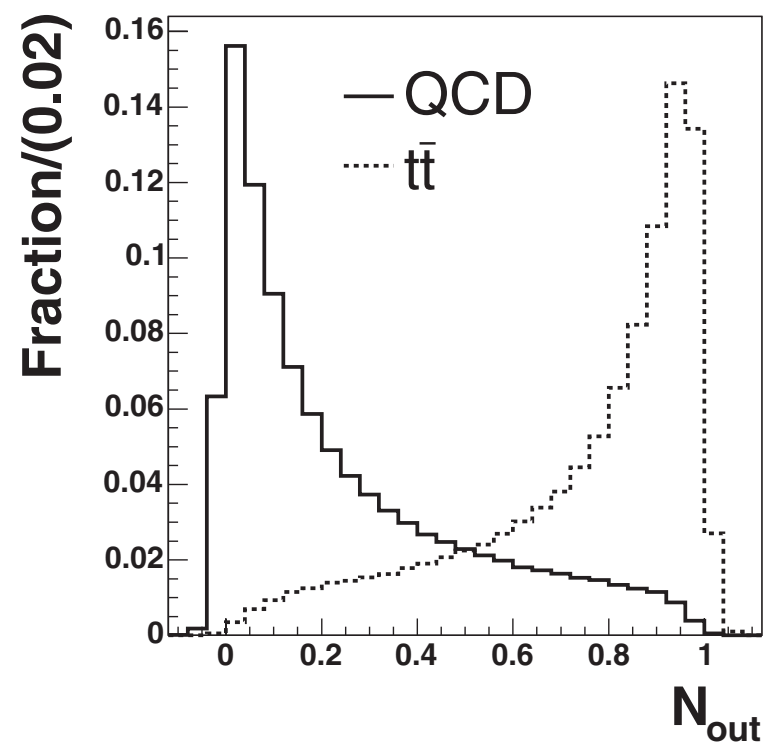

FIG. 6. Neural network output, $N_{\text {out }}$, for QCD multijet (solid histogram) and $t \bar{t}$ Monte Carlo (dashed histogram) events with $6 \leq N_{\text {jets }} \leq 8$. Histograms are normalized to unity. The neural network implementation that we use in the TMultiLayerPerceptron produces an output which is not strictly bound between 0 and 1 . the configurations investigated, the one which provides the largest expected S/B has two hidden layers with 20 and 10 hidden nodes, respectively, and 1 output node. The value of the output node, $N_{\text {out }}$, is the quantity we use as the discriminator between signal and background, as shown in Fig. 6 for the $6 \leq N_{\text {jets }} \leq 8$ sample.

\section{TAGGING $b$ QUARKS IN THE MULTIJET SAMPLE}

In order to estimate the $t \bar{t}$ content in the event sample, we exploit the heavy flavor content of $t \bar{t}$ events using a $b$-tagging algorithm based on secondary vertex reconstruction as described in detail in [22,29]. The algorithm aims at the identification of jets containing a $b$-hadron state by reconstructing its decay vertex with at least two highquality tracks with hits in the silicon vertex detector. A $b$-tagged jet ("tag," in brief) must have an associated secondary vertex with a displacement from the primary vertex in the transverse plane larger than 7.5 times the typical resolution of the vertex displacement of about $190 \mu \mathrm{m}$. Since, as shown in the next section, the background will be estimated in terms of inclusive tags rather than events, for the signal we do not consider the efficiency for tagging an event, but rather the quantity to be used in the cross section calculation is the average number of tags per event, $n_{\text {tag }}^{\text {ave }}$, which is related to the cross section using $t \bar{t}$ Monte Carlo calculations. The tagging efficiencies for jets coming from the fragmentation of $b-, c$-, or light-flavored quarks are corrected according to the efficiency seen in the

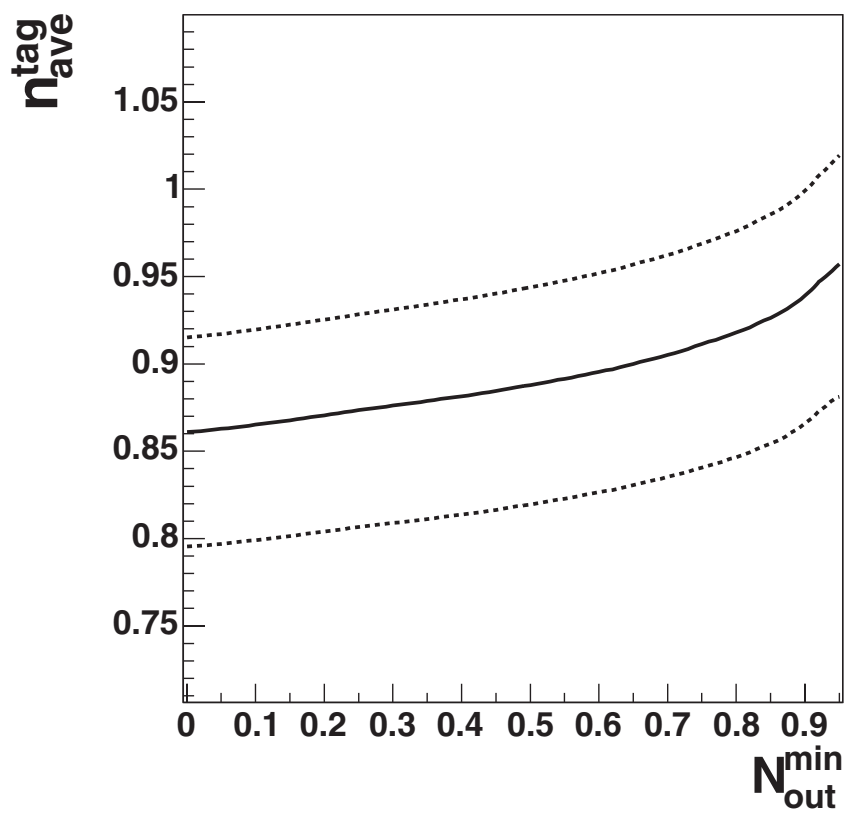

FIG. 7. Average number of tags, $n_{\mathrm{tag}}^{\mathrm{ave}}$, as a function of the threshold $N_{\text {out }}^{\text {min }}$ for $t \bar{t}$ Monte Carlo events passing the preselection and with $6 \leq N_{\text {jets }} \leq 8$. The dashed lines represent the 1- $\sigma$ uncertainty on $n_{\mathrm{tag}}^{\mathrm{ave}}$. 
data, by a factor $0.89 \pm 0.07$ for $b$ jets and $0.89 \pm 0.14$ for $c$ jets, respectively. These factors are described in detail in [22].

We find that the average number of tags present in a $t \bar{t}$ event after the preselection depends on the choice of the lower threshold, $N_{\text {out }}^{\min }$ (see Fig. 7) for the neural network output $N_{\text {out }}$. For any value of this threshold the systematic uncertainty on $n_{\text {tag }}^{\text {ave }}$ is dominated by the uncertainty of the correction factors for tagging $b$ and $c$ jets.

\section{BACKGROUND ESTIMATE}

The background for the $t \bar{t}$ multijet final state comes mainly from QCD production of heavy-quark pairs $(b \bar{b}$ and $c \bar{c}$ ) and false tags in light-quark jets. Other standard model processes such as $W / Z+$ jets have a smaller production cross section and small acceptance due to the selection cuts. Because of the current uncertainties on the Monte Carlo generation of QCD multijet events, we estimate the background from the data themselves. Given the theoretical uncertainties on the production cross section for events generated with $N$ partons at tree level, a more accurate background estimate is obtained from the data themselves rather than from theoretical prediction of cross section and simulations. The tag rate per jet is defined as the probability of tagging a jet whose tracks are reconstructed in the vertex detector. This rate is extracted from events depleted in the $t \bar{t}$ signal and is used as an estimate of the rate expected in events with different jet multiplicity. These depleted events, with exactly four jets passing the preselection ("control sample"), have $S / B \approx 1 / 3600$. This method intrinsically provides an inclusive estimate in terms of the number of tags rather than the number of tagged events. The tag rate per jet is evaluated in this control sample and is parametrized in terms of variables sensitive to both the tagging efficiency for true heavyflavored objects and the rate of false tags. These variables are the jet $E_{T}$, the number of tracks reconstructed in the vertex detector and associated to the jet, $N_{\mathrm{trk}}^{\mathrm{jet}}$, and the number of primary vertices in the event, $N_{\text {vert }}$. The tag rates per jet as a function of these variables are shown in Fig. 8 for jets with at least two tracks within the vertex detector acceptance ("fiducial" jets).

The tag rate estimates the probability that a given fiducial jet in the signal sample is tagged. Summing this probability over all fiducial jets, we obtain the expected number of tags from nonsignal processes, that is, QCD heavy- and light-flavored production altogether. Before the

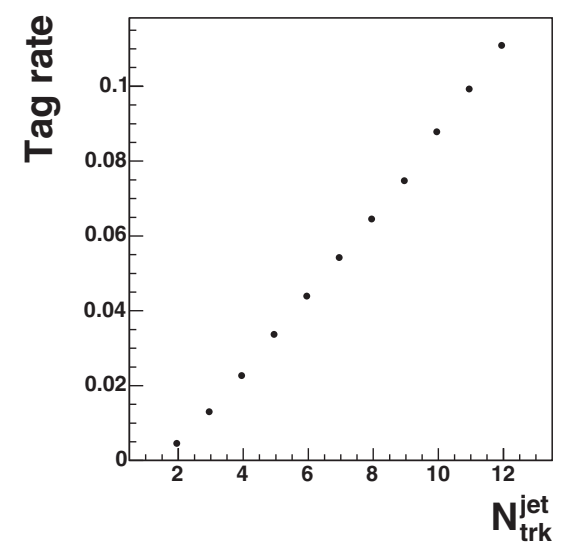

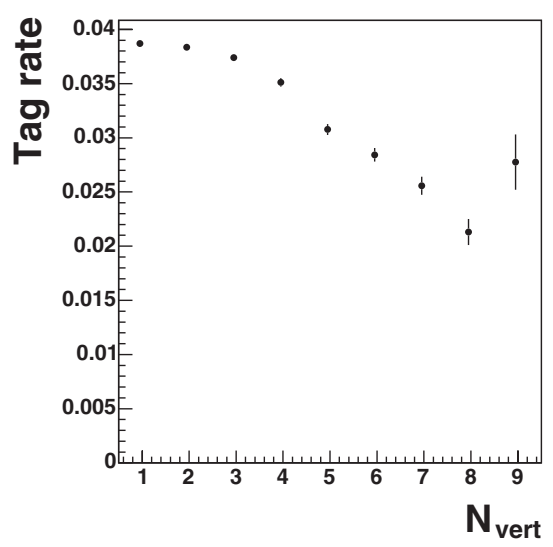

FIG. 8. Tag rate for fiducial jets as a function of jet $E_{T}, N_{\text {trk }}^{\text {jet }}$, and $N_{\text {vert }}$, as measured in the control sample with exactly four jets. 
neural-network-based kinematical selection, the multijet sample is composed essentially of background events. The accuracy of our modeling of the background processes, assuming all jets to be uncorrelated, is shown in Figs. 9-11 where we compare the $N_{\text {out }}$ distributions for tags in the data to the expected background in events with 4,5 , and 6 or more jets. The disagreement between the total number of observed and expected tags, possibly due to the presence of a small sample of $t \bar{t}$ events, amounts to no more than $0.8 \%$. This small discrepancy observed at high jet multiplicity is accounted for as a systematic uncertainty on the background estimate due to the different jet multiplicity in the signal and control samples. The kinematical selection cutting on $N_{\text {out }}$ also changes the event characteristics with respect to those found in the sample with exactly four jets, where the parametrization has been derived. This selection modifies the jet- $E_{T}$ and $\eta$ spectra so that the average tag rate per event for jets from QCD background becomes higher. However, the parametrization of the tag rate in terms of properties of the jet $\left(E_{T}\right.$ and $\left.N_{\mathrm{trk}}^{\mathrm{jet}}\right)$ is shown to describe this increase well. Residual biases due to the neural network selection are treated as systematic uncertainties on the background prediction considering, in the control sample with four jets, subsets of events with $N_{\text {out }} \geq$ $N_{\text {out }}^{\min }$ with $N_{\text {out }}^{\min }$ in the range 0.8-1.0. In this case the disagreement between the total number of observed tags and the expected background is no more than about $2.4 \%$, due to the neural network kinematical selection. The total systematic uncertainty expected on the background esti-

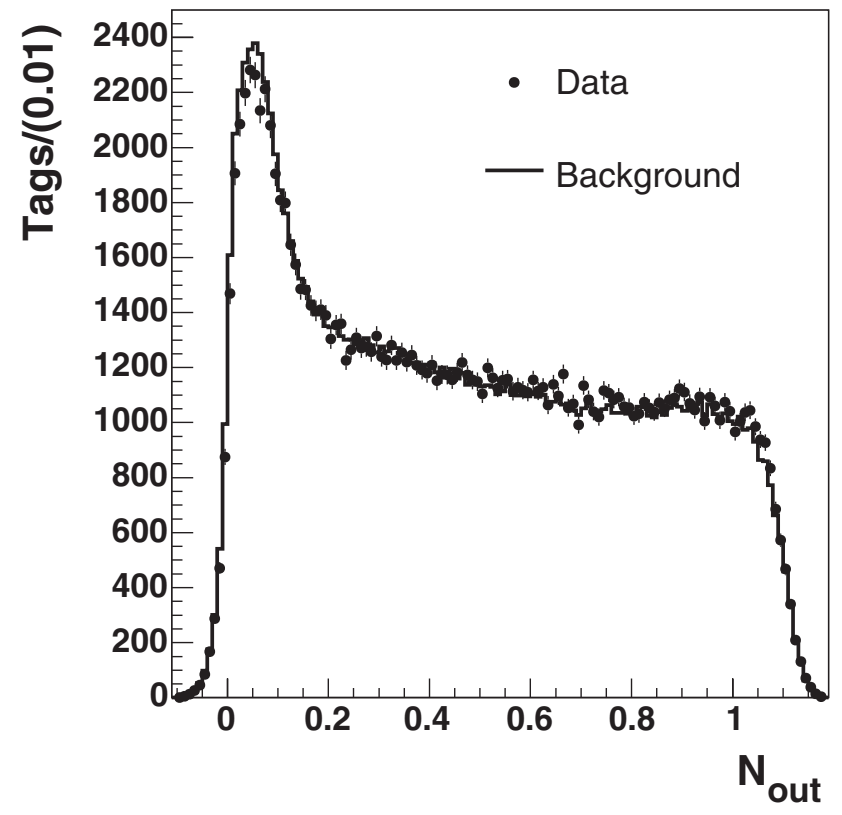

FIG. 9. $N_{\text {out }}$ distribution for tags in the data events with 4 jets, compared with the estimate from the tag rate parametrization. Events with multiple tags have multiple entries.

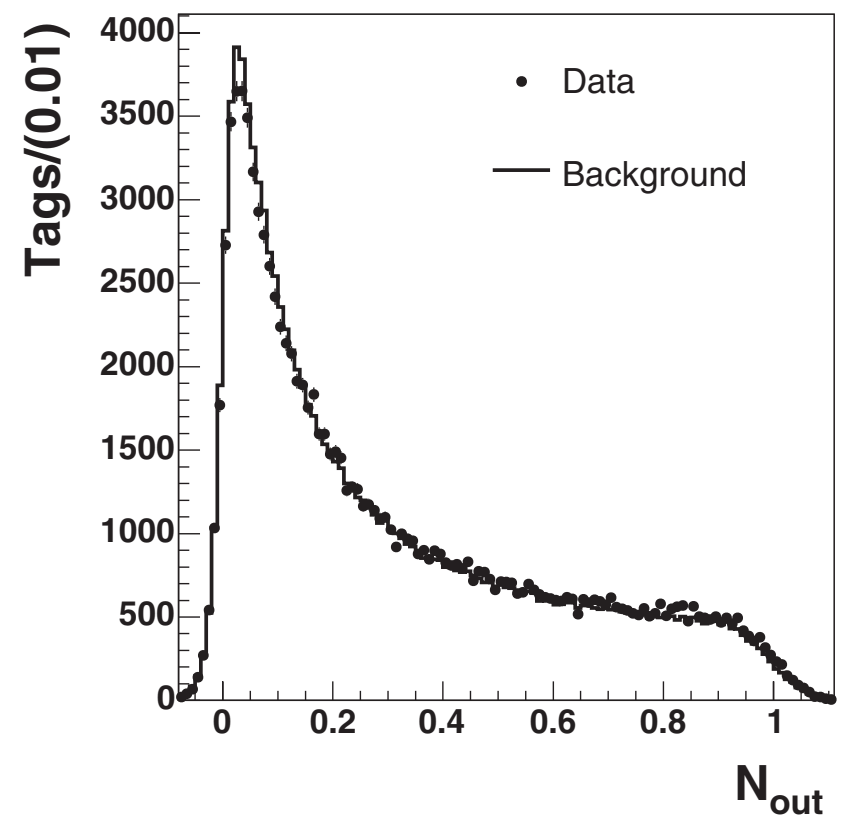

FIG. 10. $N_{\text {out }}$ distribution for tags in the data events with 5 jets, compared with the estimate from the tag rate parametrization. Events with multiple tags have multiple entries.

mate amounts then to $2.5 \%$, the quadrature sum of the two uncertainties. The contributions from running conditions, such as instantaneous luminosity and detector configuration, have been studied and found to be negligible.

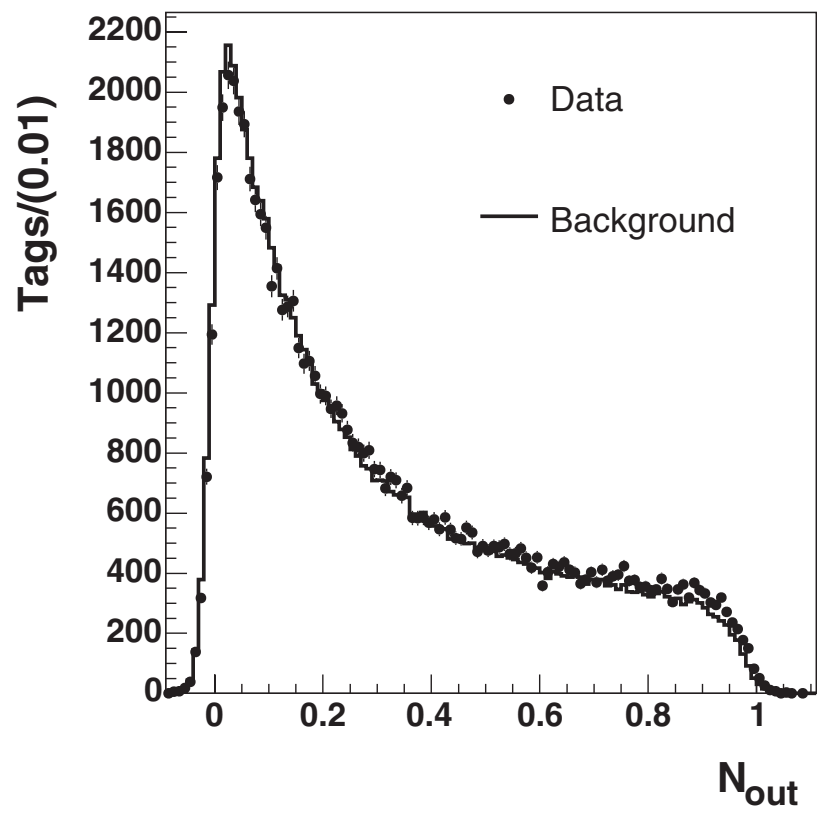

FIG. 11. $N_{\text {out }}$ distribution for tags in the data events with 6 to 8 jets, compared with the estimate from the tag rate parametrization. Events with multiple tags have multiple entries. 


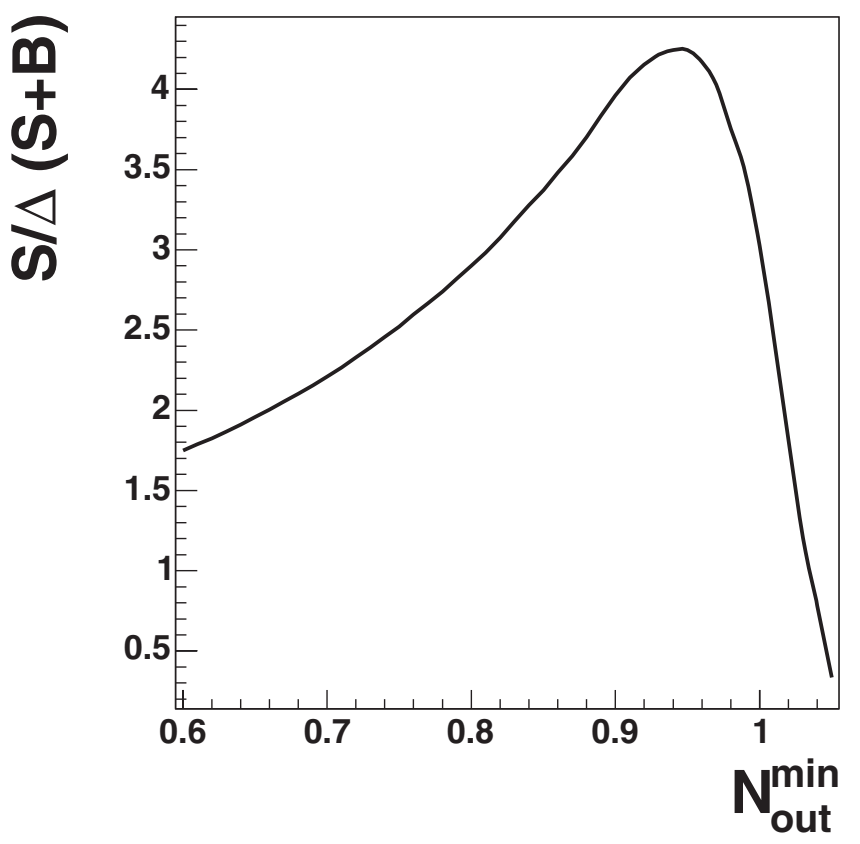

FIG. 12. Signal significance as a function of the neural network threshold $N_{\text {out }}^{\text {min }}$.

\section{OPTIMIZATION OF THE KINEMATICAL SELECTION AND ITS EFFICIENCY}

In order to obtain the most precise cross section measurement, the neural-network-based kinematical selection is optimized, in the sample $6 \leq N_{\text {jets }} \leq 8$, for the maximum signal significance for $t \bar{t}$ events, defined as the ratio between the expected signal (assuming the theoretical production cross section of $6.7 \mathrm{pb}$ ) and the total uncertainty on the sum of signal and background, where both statistical and systematic uncertainties are considered. Since we have an accurate background prediction only after the $b$-tagging requirement, the optimization refers to tagged $t \bar{t}$ events and the expected background. The cut on the neural network output which provides the maximum signal significance is $N_{\text {out }} \geq 0.94$, as can be seen in Fig. 12. Such a selection yields, before $b$ tagging, 4205 candidate events in the data with an efficiency of $4.8 \%$ for the $t \bar{t}$ signal and with S/B $\sim 1 / 12$.

The effect of the selection on $t \bar{t}$ events and on the data is summarized in Table II. The relative contribution from the

TABLE II. Efficiency of the kinematical selection measured from PYTHIA $t \bar{t}$ Monte Carlo simulation for $M_{\text {top }}=175 \mathrm{GeV} / c^{2}$ and number of events selected in the data (before requiring $b$ tagging).

\begin{tabular}{lcc}
\hline \hline Requirement & Efficiency $(\%)$ & Data \\
\hline Trigger & 58 & 4340143 \\
Preselection & 38 & 3480768 \\
$6 \leq N_{\text {jets }} \leq 8$ & 20 & 506567 \\
$N_{\text {out }} \geq 0.94$ & 4.8 & 4205 \\
\hline \hline
\end{tabular}

TABLE III. The number of events in data before and after the neural network selection, $N_{\text {out }} \geq 0.94$, for different jet multiplicities (before requiring $b$ tagging).

\begin{tabular}{lcc}
\hline \hline $\begin{array}{c}\text { Jet } \\
\text { multiplicity }\end{array}$ & $\begin{array}{c}\text { Events before } \\
\text { selection }\end{array}$ & $\begin{array}{c}\text { Events after } \\
\text { selection }\end{array}$ \\
\hline 4 & 1341622 & 118657 \\
5 & 917999 & 16157 \\
6 & 372091 & 2575 \\
7 & 109295 & 1069 \\
8 & 25181 & 561 \\
\hline \hline
\end{tabular}

leptonic channels after all the cuts is small, about 3\%. A summary of the data as a function of jet multiplicity is shown in Table III.

The systematic uncertainties affecting the $t \bar{t}$ production cross section are summarized in Table IV, with a total relative contribution of $17 \%$ on the kinematical selection efficiency. The uncertainty of $16.3 \%$ arising from the jet energy scale is dominant, since this analysis requires the presence of a large number of jets in the event which are used to build the set of kinematical variables employed in the selection. This uncertainty is evaluated by the shift in signal acceptance observed by changing the jet energy corrections within their uncertainties. Less relevant sources of uncertainty are associated with different Monte Carlo hadronization schemes (1.1\%), increased and decreased initial and final state radiation (ISR and FSR) $(2.9 \%)$, and the variation of parton distribution functions (PDFs) within their uncertainties (1.4\%). A detailed description of the procedure used to estimate these uncertainties can be found in [8]. The difference in the amount of multiple beam interactions present in the data events and in the Monte Carlo simulation is also accounted for $(2.5 \%)$.

After the kinematical selection with $N_{\text {out }} \geq 0.94$, the $b$-tagging selects 1020 events with 1233 candidate tags. The estimated background amounts to $937 \pm 30$ tags, while for $t \bar{t}$ events we expect an average number of tags $n_{\text {tag }}^{\text {ave }}=0.95 \pm 0.07$. Since the background estimate is obtained from all the events passing the selection before tagging, we need to subtract the contribution due to the $t \bar{t}$ events, as obtained from the excess of candidates with

TABLE IV. Relative systematic uncertainties on the signal efficiency, and other uncertainties related to the cross section.

\begin{tabular}{lc}
\hline \hline \multicolumn{1}{c}{ Source } & Uncertainty (\%) \\
\hline Energy scale & 16.3 \\
Parton distribution functions & 1.4 \\
Initial/Final state radiation & 2.9 \\
Monte Carlo modeling & 1.1 \\
Multiple interactions & 2.5 \\
Average number of tags & 7.4 \\
Estimated background & 2.5 \\
Integrated luminosity & 6.0 \\
\hline \hline
\end{tabular}


respect to the background, divided by $n_{\text {tag }}^{\text {ave }}$ [30]. This contribution is then subtracted from the number of events before tagging to obtain, iteratively, a new background estimate. After this correction, the number of tags expected from background sources is reduced to $846 \pm 37$ tags, where the increased uncertainty accounts for the uncertainty on $n_{\text {tag }}^{\text {ave }}$.

\section{CROSS SECTION MEASUREMENT}

In the signal region $6 \leq N_{\text {jets }} \leq 8$, the excess of observed candidate tags in the data over the background is ascribed to $t \bar{t}$ production. A measurement of the cross section can be extracted from the acceptance and the background estimate:

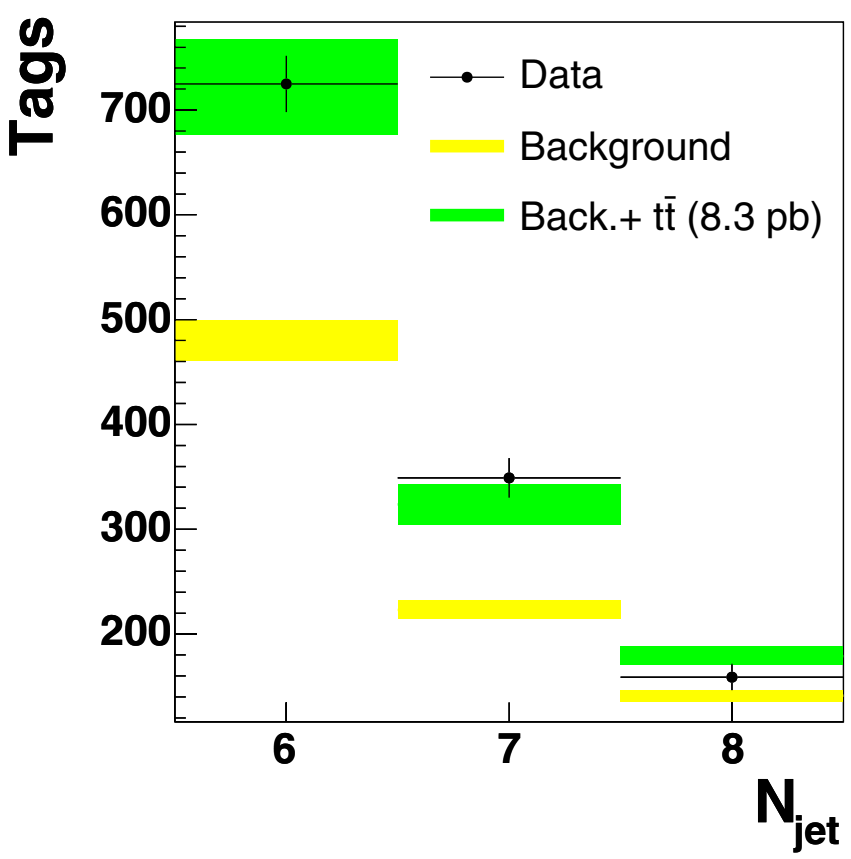

FIG. 13 (color online). Number of candidate tags as a function of the jet multiplicity for the data after the kinematical selection in the signal region, compared with the expected background. The $t \bar{t}$ expectation is based on the measured cross section of 8.3 pb. See Table V.

$$
\sigma_{t \bar{t}}=\frac{N_{\mathrm{obs}}-N_{\mathrm{bkg}}}{\epsilon_{\mathrm{kin}} \times n_{\mathrm{tag}}^{\mathrm{ave}} \times \mathcal{L}_{\mathrm{int}}}
$$

where $N_{\text {obs }}=1233$ and $N_{\text {bkg }}=846 \pm 37$ are the number of total observed and background tags, respectively, in the signal region $6 \leq N_{\text {jets }} \leq 8, \epsilon_{\text {kin }}=4.8 \pm 0.8 \%$ is the signal kinematical selection efficiency, $n_{\mathrm{tag}}^{\mathrm{ave}}=0.95 \pm 0.07$ is the average number of tags in $t \bar{t}$ events, and $\mathcal{L}_{\text {int }}=1.02 \pm$ $0.06 \mathrm{fb}^{-1}$ is the integrated luminosity of the data sample. Given the nature of our background estimate, in the above formula we use tagged jets instead of tagged events. We have verified, however, that this does not introduce any bias in the cross section, but leads to a small underestimate of the statistical uncertainty, due to the fact that some events have two or more tags. The statistical uncertainty is inflated appropriately ( $\approx+20 \%$ ), as determined from a set of Monte Carlo simulations where the number of expected signal and background events with $0,1,2$ or more tags are fluctuated according to Poisson distributions. The measured value of the $t \bar{t}$ cross section is $\sigma_{t \bar{t}}=8.3 \pm$ 1.0 (stat) ${ }_{-1.5}^{+2.0}$ (syst) \pm 0.5 (lumi) pb for a top quark mass of $175 \mathrm{GeV} / c^{2}$. In Fig. 13 the distribution of the number of observed tags and expected background is compared in the signal region to the $t \bar{t}$ signal expectation assuming the production cross section measured in this analysis. A good agreement is observed for all jet multiplicities after the kinematical selection $N_{\text {out }} \geq 0.94$, as can be seen in Table V. In addition, we show in Fig. 14 that the measured cross section is in agreement with the data over a wide $N_{\text {out }}$ range.

\section{MASS RECONSTRUCTION}

Having evaluated the $t \bar{t}$ production cross section in the preceding section, we proceed to measure the top quark mass from the same candidate events with a constrainedfitting technique. Since the kinematical selection described above is designed to have the best accuracy on the cross section measurement, we allow here for a different cut on $N_{\text {out }}$ in order to find the value which provides the best accuracy for the mass measurement. We search in simulated $t \bar{t}$ events for the $N_{\text {out }}^{\min }$ value which provides the small-

TABLE V. Observed number of tags and expected background and signal after the kinematical selection $N_{\text {out }} \geq 0.94$. The corrected background accounts for the presence of $t \bar{t}$ events before tagging. The uncertainties correspond to the quadrature sum of statistical and systematic uncertainties. See Fig. 13.

\begin{tabular}{lccccc}
\hline \hline Jet multiplicity & 4 & 5 & 6 & 7 & 8 \\
\hline Background & $16060 \pm 575$ & $2750 \pm 92$ & $536 \pm 17$ & $255 \pm 8$ & $146 \pm 5$ \\
Corrected background & $15961 \pm 677$ & $2653 \pm 112$ & $481 \pm 20$ & $223 \pm 10$ & $142 \pm 7$ \\
$t \bar{t}\left(\sigma_{t \bar{t}}=8.3 \mathrm{pb}\right)$ & $120 \pm 20$ & $266 \pm 45$ & $242 \pm 41$ & $101 \pm 17$ & $38 \pm 7$ \\
Background $+t \bar{t}$ & $16081 \pm 677$ & $2919 \pm 121$ & $723 \pm 46$ & $324 \pm 20$ & $180 \pm 10$ \\
Data & 16555 & 3139 & 725 & 349 \\
\hline \hline
\end{tabular}




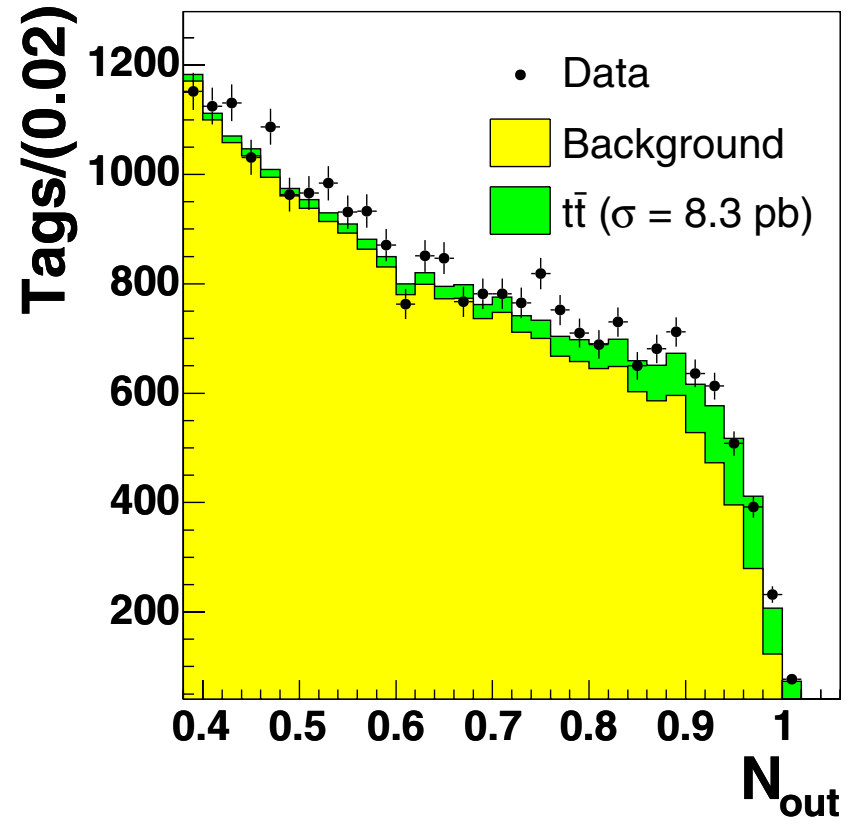

FIG. 14 (color online). $\quad N_{\text {out }}$ distribution for the tags in the data, compared with the expected background. The $t \bar{t}$ expectation is based on the measured cross section of $8.3 \mathrm{pb}$.

est statistical uncertainty expected on the mass measurement [31].

For each event we determine a reconstructed top quark mass, $m_{t}^{\text {reco }}$, from the four-momenta of the six highest- $E_{T}$ jets. This mass is entered into a distribution which is used to determine the most likely top quark mass from the data sample (see Sec. XI).

\section{Kinematical fitter}

Sixteen equations can be considered to connect the fourmomenta of the two top quarks and the six final state particles according to the $t \bar{t} \rightarrow b \bar{b} W^{+} W^{-} \rightarrow b \bar{b} q_{1} \bar{q}_{2} q_{3} \bar{q}_{4}$ hypothesis:

$$
\begin{gathered}
p_{t}^{\mu}=p_{W^{+}}^{\mu}+p_{b}^{\mu}, \\
p_{\bar{t}}^{\mu}=p_{W^{-}}^{\mu}+p_{\bar{b}}^{\mu}, \\
p_{W^{-}}^{\mu}=p_{q_{1}}^{\mu}+p_{\bar{q}_{2}}^{\mu}, \\
p_{W^{+}}^{\mu}=p_{q_{3}}^{\mu}+p_{\bar{q}_{4}}^{\mu}, \\
(\mu=0,1,2,3) .
\end{gathered}
$$

There are 13 unknown quantities, i.e., the unknown top quark mass and the three-momenta of the top quarks and of the $W$ bosons, so the kinematics of the event is overconstrained. Only the six highest- $E_{T}$ jets are used as inputs to the kinematical fitter, resulting in 90 possible permutations of two jet doublets giving a $W$ and of two jet triplets giving the top quarks. Only events with at least one $b$ tag among the six highest- $E_{T}$ jets are used in this analysis, with the association of the tagged jet to a $b$ quark, reducing the number of possible jet-to-parton permutations to 30 . We construct the $\chi^{2}$ function

$$
\begin{aligned}
\chi^{2}= & \frac{\left(m_{j j_{1}}-m_{W}\right)^{2}}{\Gamma_{W}^{2}}+\frac{\left(m_{j j_{2}}-m_{W}\right)^{2}}{\Gamma_{W}^{2}}+\frac{\left(m_{j j j_{1}}-m_{t}^{\mathrm{reco}}\right)^{2}}{\Gamma_{t}^{2}} \\
& +\frac{\left(m_{j j j_{2}}-m_{t}^{\mathrm{reco}}\right)^{2}}{\Gamma_{t}^{2}}+\sum_{i=1}^{6} \frac{\left(p_{T, i}^{\mathrm{fit}}-p_{T, i}^{\mathrm{meas}}\right)^{2}}{\sigma_{i}^{2}}
\end{aligned}
$$

where $m_{j j_{1,2}}$ are the invariant masses of the dijet systems, $m_{j j j_{1,2}}$ are the invariant masses of the trijet systems, $\Gamma_{W}=$ $2.1 \mathrm{GeV} / c^{2}$ is the measured natural width of the $W$ [32], and $\Gamma_{t}$, fixed to $1.5 \mathrm{GeV} / c^{2}$, is the assumed natural width of the top quark [33]. The measured jet transverse energies, $p_{T, i}^{\text {meas }}$, are free to vary within their known resolution, $\sigma_{i}$. The $\chi^{2}$ is minimized with respect to the seven free parameters $\left(m_{t}^{\text {reco }}\right.$, and the transverse momenta, $p_{T, i}^{\mathrm{fit}}$, of the six jets) for each of the 30 permutations of jets with final state partons. The permutation with the lowest $\chi^{2}$ is selected and a distribution ("template") of $m_{t}^{\text {reco }}$ is then formed to be used for the determination of the true top quark mass.

In order to reconstruct a data-driven background template we apply the kinematical fitter to the sample of events
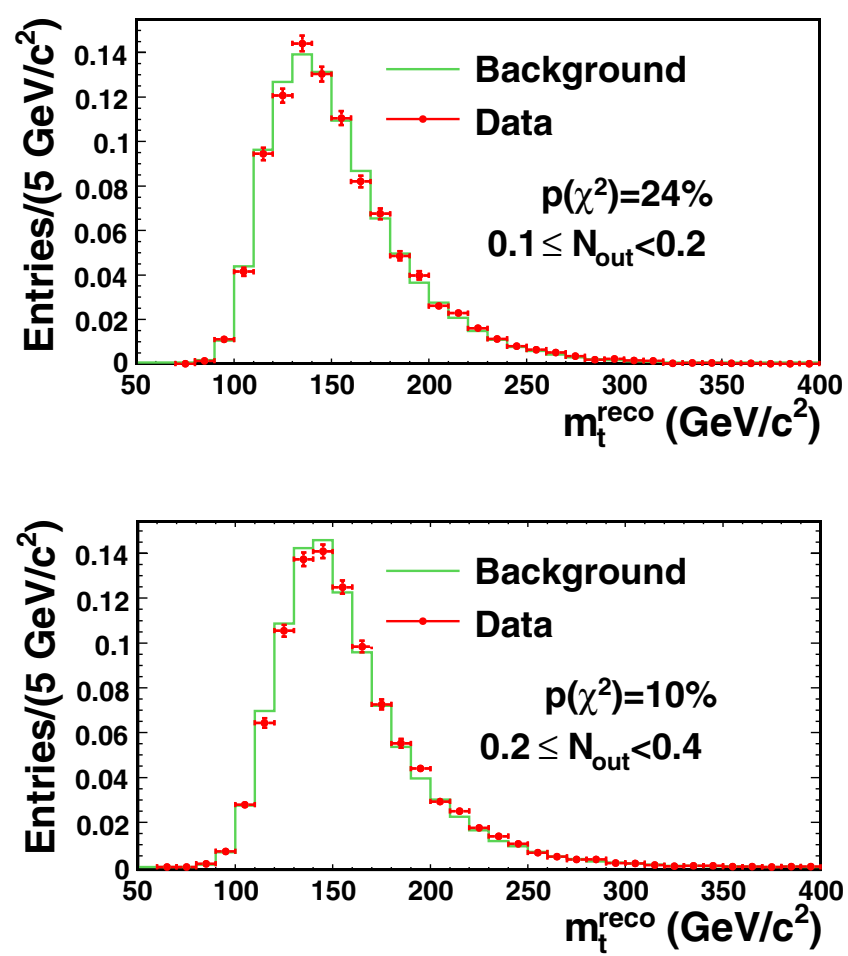

FIG. 15 (color online). Comparison of the expected versus observed $m_{t}^{\text {reco }}$. Control regions $0.1 \leq N_{\text {out }}<0.2$ (top panel) and $0.2 \leq N_{\text {out }}<0.4$ (bottom panel). The $\chi^{2}$ probability is indicated in the legend. 

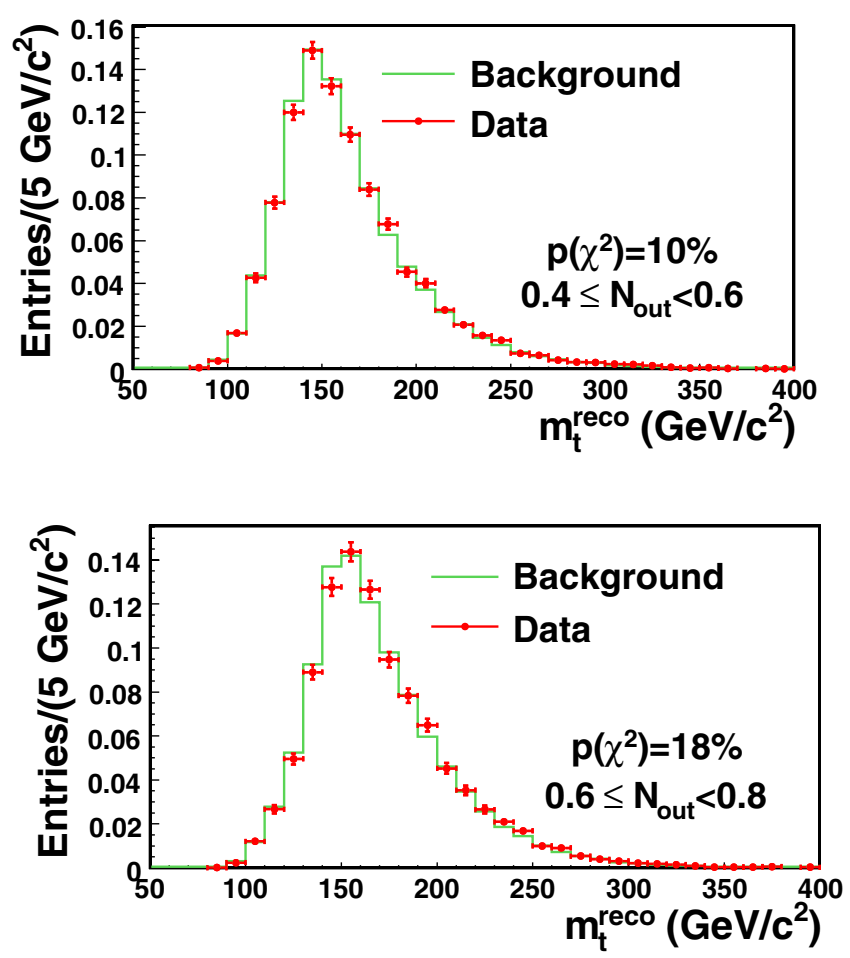

FIG. 16 (color online). Comparison of the expected versus observed $m_{t}^{\text {reco }}$. Control regions $0.4 \leq N_{\text {out }}<0.6$ (top panel) and $0.6 \leq N_{\text {out }}<0.8$ (bottom panel). The $\chi^{2}$ probability is indicated in the legend.

passing the neural network selection, but before the requirement of identified $b$ jets. Within an event the fit is performed once for each fiducial jet, assuming it is a $b$ quark. The resulting value of $m_{t}^{\text {reco }}$ enters the template with a weight given by the tag rate associated with the fiducial jet. The integral of the $m_{t}^{\text {reco }}$ distribution is the sum of all weights and represents the expected number of background tags. This procedure does not allow a separation between the background expected for events with 1 or 2 tags, but treats them together.

We follow the same approach for the data and the signal simulation, so the fit is performed for each association of tagged jets with one or the other of the two $b$ quarks; events with more than 1 tag contribute then with multiple entries. A control sample where we expect the signal to be present in fractions of few percent or below is defined by $0.1 \leq$ $N_{\text {out }} \leq 0.8$ and is further subdivided in four subregions, to check the goodness of the background modeling. As shown in Figs. 15 and 16, the background is found to describe well the $m_{t}^{\text {reco }}$ distributions in all the subsamples.

\section{LIKELIHOOD FIT}

The technique described above gives a distribution of $m_{t}^{\text {reco }}$ (one for each tag in the event) in the corresponding data sample, which is a mixture of signal and background. In order to measure the top quark mass, we compare the $m_{t}^{\text {reco }}$ distribution from the data to the signal and background templates. From the templates, we first derive probability density functions (p.d.f.'s) and then perform an unbinned likelihood fit to determine the value of true top quark mass, $\mathrm{M}_{\mathrm{top}}$, that best describes the data.

\section{A. The likelihood function}

The p.d.f.'s for the $m_{t}^{\text {reco }}$ distributions are parametrized in order to have a functional form which varies smoothly with $\mathrm{M}_{\text {top }}$. For the signal, we use $t \bar{t}$ Monte Carlo events generated with HERWIG v6.508 [34] with top quark masses ranging from 150 to $200 \mathrm{GeV} / c^{2}$ in $2.5 \mathrm{GeV} / c^{2}$ increments. The function for the signal p.d.f., $P_{\text {sig }}\left(m \mid M_{\text {top }}\right)$, represents the probability to obtain a value $m$ for $m_{t}^{\text {reco, }}$, given a true top quark mass $M_{\text {top }}$ in a $t \bar{t}$ event. The form used is a sum of a Gamma distribution, chosen to describe the reconstructed top quark mass in case of incorrect jetparton assignments, and two Gaussian distributions, which model the core of the distribution. Its explicit expression is

$$
\begin{aligned}
P_{\text {sig }}\left(m \mid M_{\text {top }}\right)= & \delta_{7} \cdot \frac{\delta_{2}^{1+\delta_{1}}}{\Gamma\left(1+\delta_{1}\right)} \cdot\left(m-\delta_{0}\right)^{\delta_{1}} \\
& \cdot \exp \left(-\delta_{2}\left(m-\delta_{0}\right)\right)+\delta_{8} \cdot \frac{1}{\sqrt{2 \pi} \delta_{4}} \\
& \cdot \exp \left(\frac{-\left(m-\delta_{3}\right)^{2}}{2 \delta_{4}^{2}}\right)+\left(1-\delta_{7}-\delta_{8}\right) \\
& \cdot \frac{1}{\sqrt{2 \pi} \delta_{6}} \cdot \exp \left(\frac{-\left(m-\delta_{5}\right)^{2}}{2 \delta_{6}^{2}}\right)
\end{aligned}
$$

where each parameter $\delta_{i}$ is linearly dependent on $\mathrm{M}_{\text {top }}$,

$$
\delta_{i}=\alpha_{i}+\beta_{i} \cdot\left(M_{\mathrm{top}}-175\right) \quad(i=0,1, \ldots, 8),
$$

so the total number of parameters used is 18 .

Figure 17 shows some of the signal templates along with their parametrized p.d.f.'s.

The background reconstructed mass is computed as described in the previous section. The integral of this distribution is the predicted amount of background tags, which is corrected for the presence of tags expected from $t \bar{t}$ events using the iterative technique described in Sec. VI. In order to account for the shape of the signal mass distribution, the correction is made by subtracting from the background distribution the residual $t \bar{t}$ contamination distributed in mass as expected for $\mathrm{M}_{\mathrm{top}}=175 \mathrm{GeV} / \mathrm{c}^{2}$. The systematic uncertainty associated with this procedure is estimated and reported in the next section. The resulting background template is parametrized with two Gamma distributions and one Gaussian distribution. The background distribution does not depend on the top quark mass. The resulting p.d.f., $P_{\text {bkd }}(m)$, is 


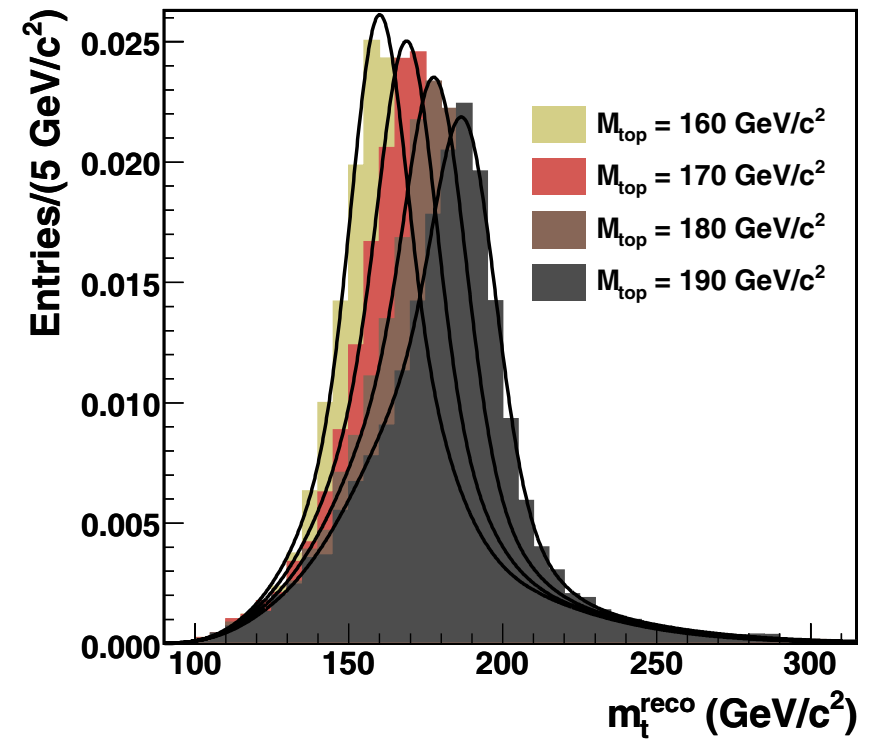

FIG. 17 (color online). Four $m_{t}^{\text {reco }}$ signal templates with their p.d.f. overlaid.

$$
\begin{aligned}
P_{\mathrm{bkd}}(m)= & \delta_{8} \cdot \frac{\delta_{2}^{1+\delta_{1}}}{\Gamma\left(1+\delta_{1}\right)} \cdot\left(m-\delta_{0}\right)^{\delta_{1}} \\
& \cdot \exp \left(-\delta_{2}\left(m-\delta_{0}\right)\right)+\delta_{9} \cdot \frac{\delta_{5}^{1+\delta_{4}}}{\Gamma\left(1+\delta_{4}\right)} \\
& \cdot\left(m-\delta_{3}\right)^{\delta_{4}} \cdot \exp \left(-\delta_{5}\left(m-\delta_{3}\right)\right) \\
& +\left(1-\delta_{8}-\delta_{9}\right) \cdot \frac{1}{\sqrt{2} \bar{\pi} \delta_{7}} \exp \left(\frac{-\left(m-\delta_{6}\right)^{2}}{2 \delta_{7}^{2}}\right)
\end{aligned}
$$

and is plotted in Fig. 18.

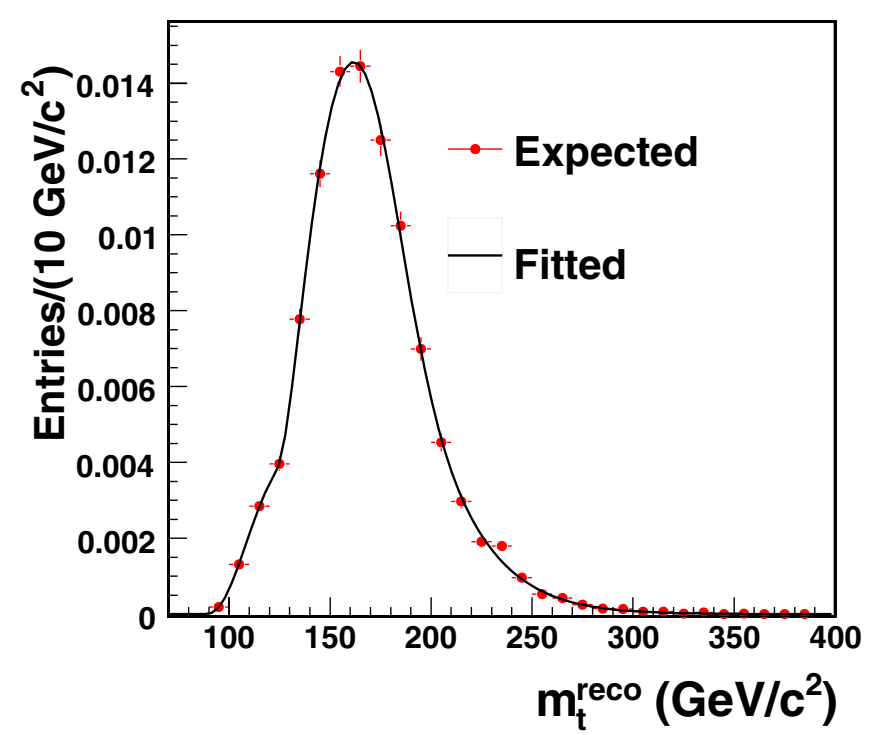

FIG. 18 (color online). $m_{t}^{\text {reco }}$ background template with its p.d.f. overlaid.
The likelihood function, $\mathcal{L}$, is constructed by assuming that the data are described by an admixture of background and $t \bar{t}$ events with a certain top quark mass. The function is obtained by multiplication of three terms. The first two terms constrain the number of background tags, $n_{b}$, to the expectation, $n_{b}^{\exp }$, and the number of signal and background tags, $n_{s}+n_{b}$, to be equal to the number observed in the data, $N$. In the third term the signal and background probabilities are assigned by comparing the measured value $m_{i}$ of $m_{t}^{\text {reco }}$ from the data with the parametrized signal and background p.d.f.'s, $P_{\text {sig }}$ and $P_{\text {bkd }}$.

$$
\begin{aligned}
\mathcal{L}= & \exp \left(-\frac{\left(n_{b}-n_{b}^{\mathrm{exp}}\right)^{2}}{2 \sigma_{n_{b}}^{2}}\right) \times \exp \left(-\frac{\left(n_{s}+n_{b}-N\right)^{2}}{2 \sigma_{N}^{2}}\right) \\
& \times \prod_{i=1}^{N} \frac{n_{s} \cdot P_{\mathrm{sig}}\left(m_{i} \mid M_{\mathrm{top}}\right)+n_{b} \cdot P_{\mathrm{bkd}}\left(m_{i}\right)}{n_{s}+n_{b}}
\end{aligned}
$$

where $\sigma_{n_{b}}$ is the expected uncertainty on the corrected background, and $\sigma_{N}=\sqrt{N}$ is the expected uncertainty on the total number of observed tags, $N$. In order to facilitate the computation, we minimize the negative logarithm of the likelihood, $-\ln \mathcal{L}$, instead of maximizing the likelihood itself. The minimization is performed with respect to the three free parameters, $n_{s}, n_{b}$, and $M_{\text {top. }}$. The statistical uncertainty on the top quark mass is taken from the points where the $-\ln \mathcal{L}$ changes by 0.5 units from its minimum.

\section{B. Verification and calibration of the method}

We investigate for possible biases in the top quark mass measurement which can be introduced by our method, and quantify its statistical power before performing the actual measurement on the data sample. We run Monte Carlo simulations of the experiment ("pseudoexperiments"), generated with the true top quark mass ranging from 150 to $200 \mathrm{GeV} / c^{2}$, and then extract the predicted amount of $m_{t}^{\text {reco }}$ values from the signal and background templates. We fix the total number of tags in each pseudoexperiment to be the same as observed in the data. Then we take our background expectation of tags passing the cuts, fluctuate it according to Gaussian statistics, and get the number of signal tags as the difference between $N$ and the fluctuated background. We perform the measurement on many different sets of Monte Carlo events in the pseudoexperiment ("pseudoevents"), and plot the fitted top quark mass with respect to the input mass in Fig. 19, seeing no systematic bias. We define the "pull" of the fit variable to be the deviation of the fitted mass from the true value in the pseudoexperiment, divided by the measurement uncertainty determined in the fit. The pull distribution is fitted with a Gaussian and its width ("pull width") indicates the accuracy of the uncertainties obtained from the fit (see Fig. 20).

The sensitivity check is performed over a range of thresholds for both $N_{\text {out }}$ and $\chi^{2}$, to achieve the smallest 


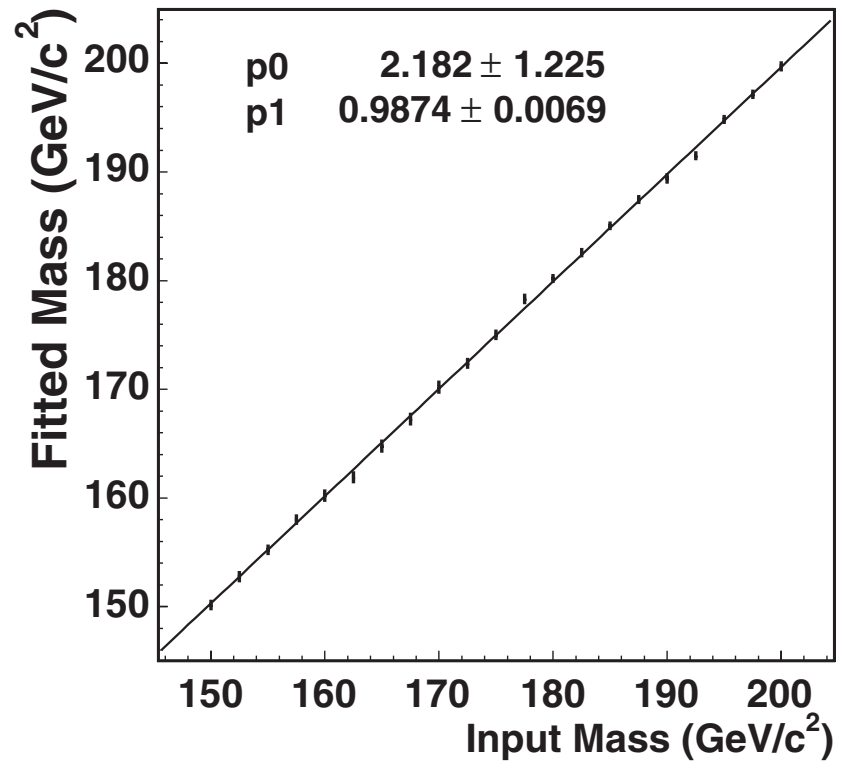

FIG. 19. Fitted mass as a function of the input top quark mass using pseudoexperiments. The fit slope is consistent with 1 . The coefficients of the fitted straight line are indicated in the legend.

expected statistical uncertainty. The $N_{\text {out }}^{\min }$ cut is varied between 0.88 and 0.96 while the upper threshold, $\chi_{\max }^{2}$, varies between 30 and 6 . The cuts for which we expect the smallest statistical uncertainty on the mass measurement are $N_{\text {out }} \geq 0.91$ and $\chi^{2} \leq 16$. The $\chi^{2}$ cut improves the background rejection so that we need to apply a threshold on $N_{\text {out }}$ lower than that used for the cross section measurement.

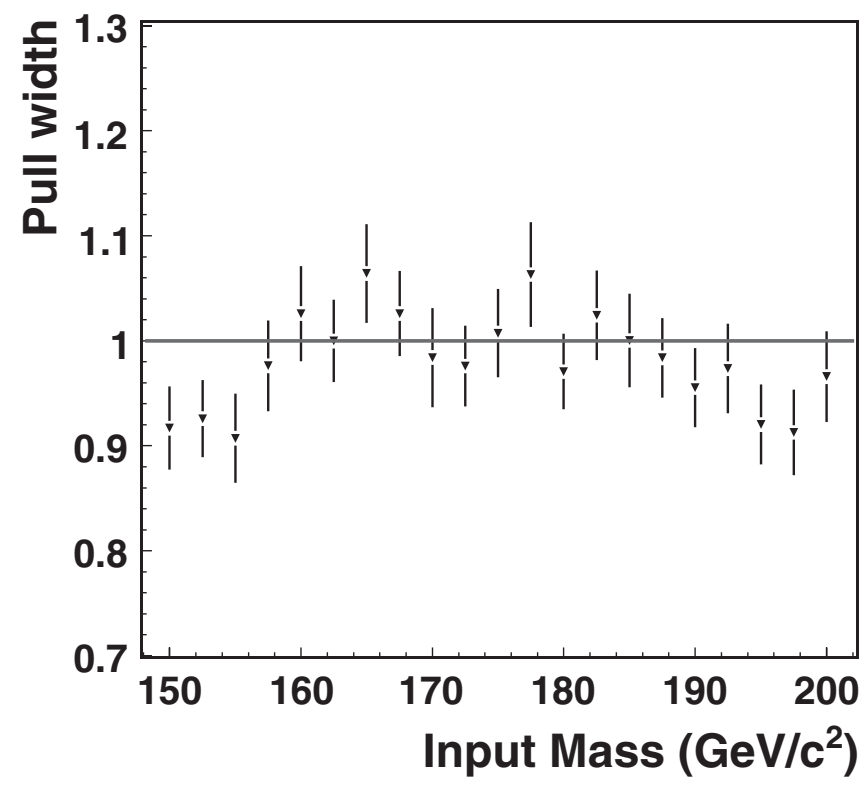

FIG. 20. Width of the pull distributions, for different top quark input masses.

\section{SYSTEMATIC UNCERTAINTIES ON THE TOP QUARK MASS}

Various sources of systematic uncertainty affect the top quark mass measurement. Systematic effects arise from mismodeling in the simulation of the detector response to jets, and from uncertainties in the simulation of the $t \bar{t}$ signal. The evaluation of the mass shift due to each source of systematic uncertainty consists in generating pseudoexperiments varying the effects from each possible source by $\pm 1 \sigma$, building new templates out of the varied sample, and determining how much the fitted top quark mass shifts. We take as an estimate of the respective uncertainty the resulting half-difference between the extreme values of the measured mass. If the shift is smaller than the statistical uncertainty on the estimate itself, we assign the latter as the systematic uncertainty.

The main contribution to the systematic uncertainty stems from the residual uncertainty on the jet energy after it is corrected for the known effects [23]. These include calorimeter nonlinearity in response to single hadronic particles, energy loss into noninstrumented calorimeter regions, energy added to the jet by secondary interactions and the underlying event, and energy lost outside the jet cone. We calculate the systematic uncertainty originating from each of these sources varying each corrected jet energy in the simulation by the corresponding uncertainty and performing pseudoexperiments with the modified resulting templates, and finally adding the observed mass shifts in quadrature to quote a total systematic due to the jet energy scale. Since the jet energy corrections are derived on data samples deprived of heavy quarks, we add an additional uncertainty of the order of $0.6 \%$ evaluated considering the different fragmentation properties of $b$ quarks [7]. As done for the generic jet energy scale uncertainties, we perform pseudoexperiments where we vary the $b$-jet energy scale accordingly and use half the variation in the fitted top quark mass as the $b$-jet energy scale uncertainty.

Many sources of systematic effects arise from uncertainty in the Monte Carlo modeling of the hard interaction. PYTHIA and HERWIG generators differ in their hadronization schemes and in their description of the underlying event and multiple interactions. A corresponding systematic uncertainty is evaluated drawing top quark reconstructed masses from PYTHIA-generated events and comparing the resulting mass distributions with the template constructed using HERWIG. Additional jets coming from ISR and FSR might fall among the six leading jets and populate the tails in the $m_{t}^{\text {reco }}$ distribution. These effects are studied using pseudoexperiments where we extract top quark reconstructed masses from templates generated with different values of $\Lambda_{\mathrm{QCD}}$ and scale factor $K$ [10], and comparing them with the standard templates. Since the shift is very small, we assign the statistical uncertainty on the shift to be the ISR/FSR systematic uncertainty. 
The choice of PDF inside the proton affects the kinematics of $t \bar{t}$ events and thus possibly the top quark mass measurement. We estimate the uncertainty from the difference in top quark mass resulting from the use of Monte Carlo samples based on the default CTEQ5L [35] PDF and on the ones calculated from the MRST group [36], MRST72 and MRST75, which differ by the value of $\Lambda_{\mathrm{QCD}}$ used to compute the PDF.

The background normalization is known to 5\% from the tag rate parametrization technique. We vary the background contribution using pseudoexperiments where we increase or decrease the expected background amount by its uncertainty.

We consider also the uncertainty associated with the small presence of signal in the data-driven background. To do so we build two background templates where we subtract from the background mass distribution the expected signal mass distribution assuming the two values $172.5 \mathrm{GeV} / c^{2}$ and $177.5 \mathrm{GeV} / c^{2}$ for the top quark mass. We reconstruct the top quark mass from the two background templates and take the difference in the results as the uncertainty associated to this effect. A systematic uncertainty due to the finite size of the Monte Carlo samples used to build the mass templates is determined by varying each of the template bin entries randomly, consistent with a Poisson distribution, creating 100 such new templates, reparametrizing them to determine as many fitted top quark masses. The width of the resulting distribution is used as the systematic uncertainty.

A bias in the measurement can arise if an inadequate functional form is used for the $m_{t}^{\text {reco }}$ templates. This is checked performing pseudoexperiments where we extract mass values directly from the $m_{t}^{\text {reco }}$ histograms and compare them with the parametrized p.d.f.'s. The average of the difference between the fitted top quark masses and the input masses is chosen as a systematic uncertainty on the functional parametrization. The $b$-tagging efficiency agrees well between data and simulation; still a possible

TABLE VI. Breakdown of systematic uncertainties from different sources.

\begin{tabular}{lc}
\hline \hline Source & Uncertainty $\left(\mathrm{GeV} / c^{2}\right)$ \\
\hline Jet energy scale & 4.5 \\
Generator & 1.0 \\
$b$-jet energy scale & 0.5 \\
Parton distribution function & 0.5 \\
Background shape & 0.5 \\
Background fraction & 0.5 \\
ISR & 0.5 \\
FSR & 0.5 \\
$b$ tag & 0.5 \\
MC statistics & 0.1 \\
Template parametrization & 0.1 \\
Total & 4.8 \\
\hline \hline
\end{tabular}

dependence on jet kinematical properties could lead to a shift in the measured mass. We evaluate here a systematic due to $E_{T}$ dependence of $b$-tagging scale factors, allowing for a slope on the $E_{T}$ dependence (consistent at $1 \sigma$ with the measurements) and determining the shift in the fitted top quark mass using the modified templates. Since the background estimate is data-driven, the analysis is sensitive to an overall uncertainty in the $b$-tagging scale factor only through signal shapes.

Table VI shows a summary of all the systematic uncertainties; the total systematic uncertainty amounts to $4.8 \mathrm{GeV} / c^{2}$.

\section{MASS MEASUREMENT}

After the kinematical selection with $N_{\text {out }} \geq 0.91$, the $b$-tagging requirement, and the cut on the goodness of the fit, $\chi^{2} \leq 16$, we find 926 tags in 772 events. The background, corrected as in the cross section measurement for the contamination of $t \bar{t}$ events (see Sec. VI), amounts to $567 \pm 28$ tags.

The likelihood fit is applied to the data sample to derive $M_{\text {top }}=174.0 \pm 2.2$ (stat) \pm 4.8 (syst) $\mathrm{GeV} / c^{2}$. The plot in Fig. 21 shows the fitted top quark mass distribution for the data compared to the expected background and the signal for a top quark mass of $174.0 \mathrm{GeV} / c^{2}$. The plot in Fig. 22 compares the measured statistical uncertainty with the expected distribution from pseudoexperiments using as input mass $M_{\text {top }}=174.0 \mathrm{GeV} / c^{2}$. We find that the $p$ value for our statistical uncertainty is $40 \%$. As for the case of the

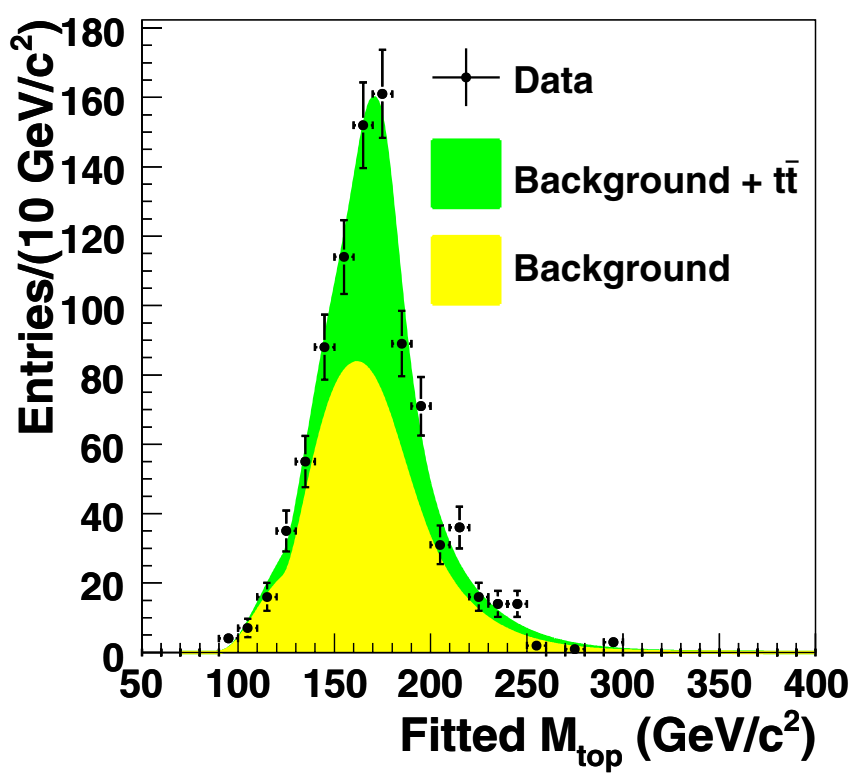

FIG. 21 (color online). The fitted top quark mass distribution for events with $N_{\text {out }} \geq 0.91, \chi^{2} \leq 16$, and at least $1 b$-tagged jet. Superimposed are the background and the $t \bar{t}$ signal expected for $M_{\text {top }}=174.0 \mathrm{GeV} / c^{2}$. 


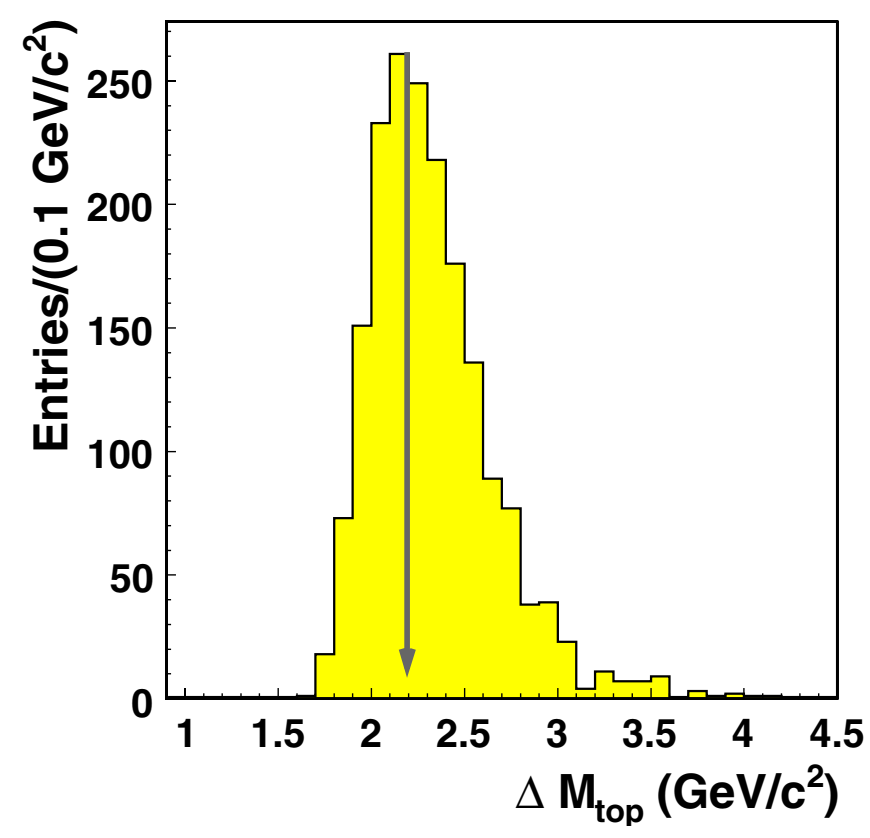

FIG. 22 (color online). The expected statistical uncertainty using pseudoexperiments with input top quark mass equal to 174.0 GeV $/ c^{2}$ for the $t \bar{t}$ signal. The arrow represents the measured statistical uncertainty.

cross section measurement, Monte Carlo simulations indicate the need to increase the statistical uncertainties by $5 \%$ to account for the use of multiple entries, one for each tag in the same event, and their correlations.

As a last check, we perform the measurement removing from the likelihood definition the Gaussian term which constrains the number of background tags to be as predicted via the tag rate parametrization, and we obtain nearly the same value, $M_{\text {top }}=174.1 \pm 2.2$ (stat) $\mathrm{GeV} / c^{2}$.

The measurement presented here is the most precise measurement to date of the top quark mass in the allhadronic decay channel. The result is consistent with the measurements obtained in the same channel at $\sqrt{s}=$ $1.8 \mathrm{TeV}$ [6], with the measurement obtained at $\sqrt{s}=$ $1.96 \mathrm{TeV}$ using $\mathcal{L}_{\text {int }}=311 \mathrm{pb}^{-1}$ of data with a different technique [5], and with the results obtained in the other channels by the CDF [10] and D0 [13] Collaborations.

\section{CONCLUSIONS}

Using an optimized neural-network-based kinematical selection and a $b$-jet identification technique, we are able to improve the S/B of the initial multijet sample obtained with a dedicated trigger from 1 in 1100 to approximately 1 in 2 . With the selected sample, we measure the $t \bar{t}$ production cross section to be $\sigma_{t \bar{t}}=8.3 \pm 1.0(\text { stat })_{-1.5}^{+2.0} \times$ (syst) \pm 0.5 (lumi) pb assuming $M_{\text {top }}=175 \mathrm{GeV} / c^{2}$, improving the previous measurement [37] in the same channel [4]. These results agree well with the standard model expectation of $\sigma_{t \bar{t}}=6.7 \mathrm{pb}$ for the same value of the top quark mass and with the results obtained in the leptonic channels. Using a slightly modified selection we reconstruct the top quark invariant mass for the overconstrained kinematical system and compare it to parametrized templates representing signal and background. A likelihood fit is used to measure a top quark mass of $M_{\text {top }}=174.0 \pm$ 2.2 (stat) \pm 4.8 (syst) $\mathrm{GeV} / c^{2}$, which improves the previous measurement [38] in the same channel [5], and agrees well with the results obtained in the leptonic channels.

\section{ACKNOWLEDGMENTS}

We thank the Fermilab staff and the technical staffs of the participating institutions for their vital contributions. This work was supported by the U.S. Department of Energy and National Science Foundation; the Italian Istituto Nazionale di Fisica Nucleare; the Ministry of Education, Culture, Sports, Science and Technology of Japan; the Natural Sciences and Engineering Research Council of Canada; the National Science Council of the Republic of China; the Swiss National Science Foundation; the A.P. Sloan Foundation; the Bundesministerium für Bildung und Forschung, Germany; the Korean Science and Engineering Foundation and the Korean Research Foundation; the Particle Physics and Astronomy Research Council and the Royal Society, U.K.; the Russian Foundation for Basic Research; the Comisión Interministerial de Ciencia y Tecnología, Spain; in part by the European Community's Human Potential Programme under Contract No. HPRNCT-2002-00292; and the Academy of Finland.
[1] Z. J. Xiao, J. Y. Zhang, L. D. Wan, X. L. Wang, and G. R. Lu, J. Phys. G 21, 19 (1995).

[2] M. Cacciari et al., J. High Energy Phys. 04 (2004) 068; N. Kidonakis and R. Vogt, Phys. Rev. D 68, 114014 (2003).

[3] G. L. Kane and S. Mrenna, Phys. Rev. Lett. 77, 3502 (1996).

[4] A. Abulencia et al. (CDF Collaboration), Phys. Rev. D 74,
072005 (2006).

[5] T. Aaltonen et al. (CDF Collaboration), Phys. Rev. Lett. 98, 142001 (2007).

[6] F. Abe et al. (CDF Collaboration), Phys. Rev. Lett. 79, 1992 (1997); B. Abbott et al. (D0 Collaboration), ibid. 83, 1908 (1999).

[7] A. Abulencia et al. (CDF Collaboration), Phys. Rev. Lett. 
97, 082004 (2006).

[8] A. Abulencia et al. (CDF Collaboration), Phys. Rev. D 74, 072006 (2006).

[9] D. Acosta et al. (CDF Collaboration), Phys. Rev. Lett. 96, 202002 (2006).

[10] A. Abulencia et al. (CDF Collaboration), Phys. Rev. D 73, 092002 (2006); 74, 032009 (2006).

[11] V. M. Abazov et al. (D0 Collaboration), Phys. Lett. B 626, 35 (2005); 626, 45 (2005); 626, 55 (2005).

[12] V.M. Abazov et al. (D0 Collaboration), Fermilab Report No. FERMILAB-PUB-06-426-E.

[13] V. M. Abazov et al. (D0 Collaboration), Phys. Rev. D 74, 092005 (2006).

[14] D. Acosta et al. (CDF Collaboration), Phys. Rev. D 71, 032001 (2005).

[15] We use a cylindrical coordinate system where $\theta$ is the polar angle to the proton beam direction at the event vertex, $\phi$ is the azimuthal angle about the beam axis, and pseudorapidity is defined $\eta=-\ln \tan (\theta / 2)$. We define transverse energy as $E_{T}=E \sin \theta$ and transverse momentum as $p_{T}=p \sin \theta$, where $E$ is the energy measured in the calorimeter and $p$ is the magnitude of the momentum measured by the spectrometer.

[16] L. Balka et al., Nucl. Instrum. Methods Phys. Res., Sect. A 267, 272 (1988).

[17] M. Albrow et al., Nucl. Instrum. Methods Phys. Res., Sect. A 480, 524 (2002).

[18] G. Ascoli et al., Nucl. Instrum. Methods Phys. Res., Sect. A 268, 33 (1988).

[19] D. Acosta et al., Nucl. Instrum. Methods Phys. Res., Sect. A 494, 57 (2002).

[20] F. Abe et al. (CDF Collaboration), Phys. Rev. D 45, 1448 (1992).

[21] F. Abe et al. (CDF Collaboration), Phys. Rev. Lett. 68, 1104 (1992).

[22] D. Acosta et al. (CDF Collaboration), Phys. Rev. D 71, 052003 (2005).
[23] A. Bhatti et al., Nucl. Instrum. Methods Phys. Res., Sect. A 566, 375 (2006).

[24] The missing transverse energy $\vec{E}_{T}$ is calculated as the vector sum of the energy in each calorimeter tower multiplied by a unit vector in the azimuthal direction of the tower. If isolated high momentum muons are found in the event, the $\vec{E}_{T}$ is corrected by subtracting the muon energy in the calorimeter and adding the muon $p_{T}$ to the vector sum. $\not{E}_{T}$ is defined as the magnitude of $\vec{E}_{T}$.

[25] MLPFIT: A Tool for Multi-Layer Perceptrons, http:// schwind.home.cern.ch/schwind/MLPfit.html.

[26] R. Brun and F. Rademaker, ROOT Version 4.00/08, http:// root.cern.ch/.

[27] F. Abe et al. (CDF Collaboration), Phys. Rev. D 51, 4623 (1995).

[28] T. Sjöstrand et al., Comput. Phys. Commun. 135, 238 (2001).

[29] A. Abulencia et al. (CDF Collaboration), Phys. Rev. D 73, 032003 (2006).

[30] F. Abe et al. (CDF Collaboration), Phys. Rev. D 50, 2966 (1994).

[31] The systematic uncertainty expected on the mass measurement is rather insensitive to the neural net cut and has been neglected in the choice of the best performing value of $N_{\text {out }}^{\text {min }}$

[32] W.-M. Yao et al. (Particle Data Group), J. Phys. G 33, 1 (2006).

[33] S. M. Oliveira, L. Brucher, R. Santos, and A. Barroso, Phys. Rev. D 64, 017301 (2001).

[34] G. Marchesini et al., Comput. Phys. Commun. 67, 465 (1992); G. Corcella et al., J. High Energy Phys. 01 (2001) 010.

[35] H. L. Lai et al., Eur. Phys. J. C 12, 375 (2000).

[36] A.D. Martin, R. G. Roberts, W. J. Stirling, and R.S. Thorne, Eur. Phys. J. C 4, 463 (1998).

[37] $\sigma_{t \bar{t}}=7.5 \pm 2.1$ (stat) ${ }_{-2.2}^{+3.3}$ (syst) ${ }_{-0.4}^{+0.5}$ (lumi) pb.

[38] $M_{\text {top }}=177.1 \pm 4.9$ (stat) \pm 4.7 (syst) $\mathrm{GeV} / c^{2}$. 